\title{
Scientific Computing Challenges Arising From Space-Borne Observations
}

\author{
MILTON HALEM
}

Invited Paper

Supercomputing simulation in conjunction with the autonomous acquisition of space-borne sensor data have led to dramatic contributions in our understanding of the physical processes occurring in the space and earth sciences over the past quartercentury. Future plans and commitments for both these technologies suggest continued exponential growth over the next decade in computing power and satellite data acquisition. As a result, a new emphasis on multidisciplinary research is emerging, creating unprecedented scientific challenges to exploit these technologies. Illustrative of these challenges, scientific results of modeling computations are presented from a variety of disciplines that have greatly benefited from both the accessibility of supercomputers and the availability of satellite data. The five following supercomputing problems are described: 1) determining the motions of the earth's inner core boundary from outer space measurements of the magnetic field, 2) simulating the global dynamics of the earth's ocean currents, sea-ice cycles, land surface biology, and interannual atmospheric climates, 3) upper atmospheric chemistry, 4) modeling solar flare eruptions, and 5) the role of jet processes in the birth of stars. In addition, two examples indicating the growing importance of the use of supercomputers for scientific visualization are presented. The first deals with remote mapping from space of the earth's topography or other planets. The second problem from astrophysics deals with mapping the cosmic background radiation in the presence of other astronomical signatures. Finally a brief overview of interdisciplinary modeling plans under development for the next generation of supercomputers is given.

\section{INTRODUCTION}

During the past quarter-century, we have witnessed the birth and maturation of two technological revolutions that together have profoundly influenced the evolution of terrestrial, atmospheric, planetary, solar, space plasma, and astrophysical sciences. Supercomputing is the first of these technologies-with exponential growth in computational power [Fig. 1(a)] enabling simulation research to emerge as a new and valuable scientific methodology. Robotic spaceborne sensing and exploration, made possible by the advent of space flight, is the second technology-with exponential growth of space science data [Fig. 1(b)] providing the basis for these scientific advances and leading to a new emphasis on interdisciplinary research. The exploitation of these two

Manuscript received January 18, 1989; revised March 10, 1989. The author is with the Space Data and Computing Division, NASA/Goddard Space Flight Center, Greenbelt, MD 20771, USA. IEEE Log Number 8928431. new technologies has already produced a wealth of scientific discoveries and insights that are helping explain the mechanisms that govern physical processes occurring on earth and in deep space.

In particular, supercomputer access has provided earth and space scientists with a unique and powerful new tool as potentially important to their research as were the development of telescopes and microscopes. Through the use of complex numerical models, scientists are realistically simulating the behavior of nonlinear physical processes occurring in nature that cannot be duplicated in the traditional laboratory environment nor addressed by conventional theoretical analysis. For example, detailed simulation studies are being used to explore parametrically the nonlinear effects in the evolution of systems under idealized conditions that normally would take climatic, geologic, or astrophysical time scales. Other applications of simulation studies are leading to the design of new and improved sensors and observing systems for the continuous global monitoring of the earth and space environment.

Similarly, space data acquired by advanced robotic sensors have provided scientists with rare views of dynamic global processes of the near or distant environments that have never before been revealed. For example, the recent availability of powerful interactive computers enabled retrospective processing of space observations that produced the first global view of the earth's biosphere (Fig. 2) and the zodiacal view of our galaxy in the infrared (Fig. 3). Such panoramic views of the earth and the sky could not have been acquired from ground-based observatories with the simultaneity and continuity of coverage afforded by spaceobserving systems. The confluence of the great space observatories such as the Hubble Space Telescope, the Advanced X-Ray Astrophysics Facility, and the Earth Observing System planned for space flight in the 1990s in conjunction with the next generation of supercomputers will offer even more exciting scientific challenges to understanding the physical processes taking place in the earth system, the solar-terrestrial environment, and the far reaches of the universe.

In this regard, the general objective of this paper is to present, within the context of the theme of this ProceEdings 




(a)

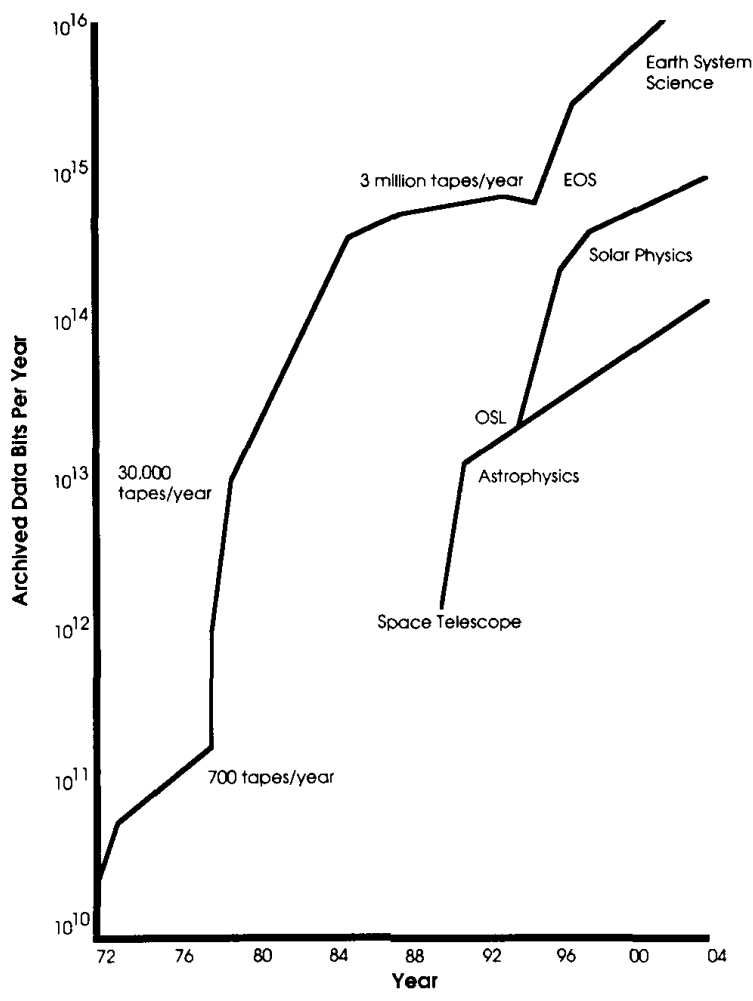

(b)

Fig. 1. (a) Growth of computer speed and memory requirements. (b) Growth in archived data.

OF THE IEEE, typical examples of the exciting scientific problems arising in space and earth sciences that have made successful use of supercomputing. For this purpose, we chose the following three problems to illustrate the ties between space observations and modeling: 1 ) inferring the motions of the inner core of the solid earth through observations of the magnetic fields surrounding the earth, 2) simulating dynamics of solar flare eruptions from solar magnetic measurements related to the convective processes internal to the sun, and 3) modeling recently discovered stellar jet stream observations associated with regions of active star formation. In addition, we present the most recent advances in supercomputing modeling applications dealing with the dynamic behavior of the atmosphere, ocean, cryosphere, biosphere, and chemistry of the upper atmosphere. Finally, to show the growing influence of new trends in supercomputing technology on these sciences, two applications are presented. The first, making use of massively parallel processing for mapping the topography, and the second, from astrophysics, making use of interactive image visualization for cosmic background radiation mapping.

The specific results derived from these scientific problems are described in Section II and are intended to provide the reader with sufficient detail to grasp the scientific and computational complexities of the subject. Clearly, arriving at a realistic depth in so many subjects in so short a review meant sacrificing a self-contained presentation of the technical material. We attempted to employ a minimum of discipline jargon-perhaps overstepping bounds in some cases-but leave the reader the choice to peruse or skip over the material when it gets too detailed. A brief scientific problem motivation, written for the layperson, is provided as introduction to each discipline problem. For those readers with scientific backgrounds in related disciplines, it is hoped that an appreciation can be gained for common supercomputing experiences facing investigators using similar algorithmic schemes.

A secondary motive in selecting these problems was to indicate the relationships between future space missions in these sciences and the way they are supporting the thrust toward multidisciplinary modeling research. Section III briefly sketches the classes of interdisciplinary research just getting started and their data requirements. This section projects the direction of the problems described in Section II.

While these supercomputing applications have shown remarkable success in the limited examples presented, adequate resources are sorely lacking to implement them with the full physical complexities required. In recognition of the future requirements, not just in the space and earth sciences, but occurring in the aerosciences as well, the NASA Office of Aeronautics and Space Technology has proposed an extensive 5-year program calling for a high-performance computing initiative $(\mathrm{HPCl})$. $\mathrm{HPCl}$ is also being coordinated with other federal agencies as part of an even broader national computing effort to maintain this country's leadership position in the supercomputing field. NASA's participation is directed toward meeting the grand scientific computing challenges described herein.

\section{Earth and Space Science Modeling}

In the following sections we briefly present the observational background and some results of selected modeling efforts representing the current state of the art in earth modeling, earth systems modeling, image modeling for remote sensing science, modeling in solar physics, interactive visualization, and simulation of astrophysical phenomena. The studies are but a small sample of the numerous models investigating the many aspects of physical phenomena occurring in these disciplines. The examples were partly selected because of their relevant applications to observational programs from space.

However, in using such observations, space scientists are being forced to deal more and more with a unique class of 




Fig. 2. Global pattems of biological productivity-in essence hand and ocean vegetation. Land pattems are detemined from chlorophyll measurements taken from the NOAA-7 polar orbiting satellite, and ocean patterns from the NASA Nimbus 7 satellite. Ocean productivity patterns represent an average over 18 months and range from red cosost productive to purble fleast productivel Land patferns represent the potential productivity averaged over 3 years and range trom deep green representing rain forests, to beige representing deserts and barren regions. This map was produced by the Space Data and Connputing Division, NASA/Goddard Space Fight Center.

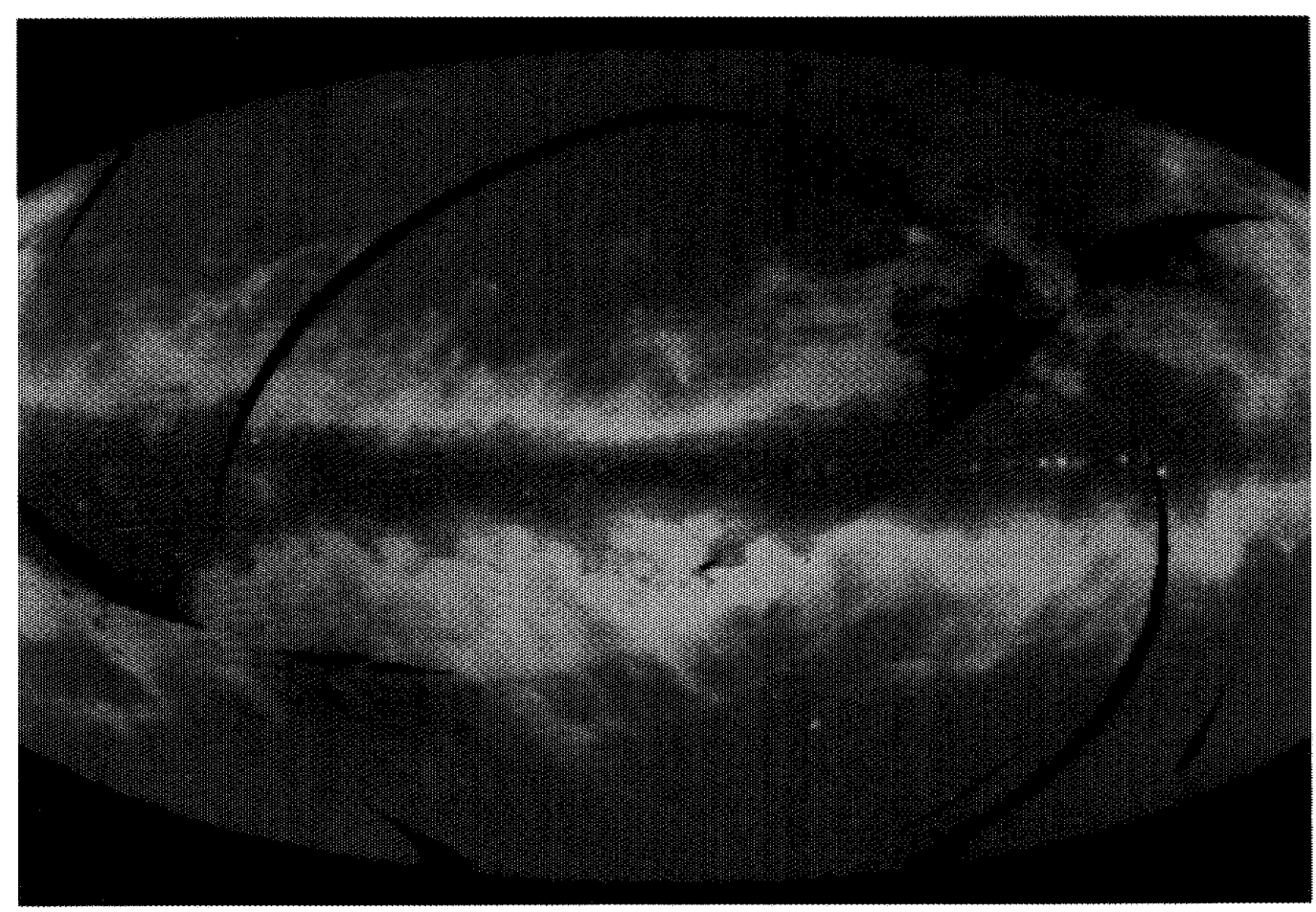

Fig. 3. Infrared astronomical satelite IRAS time-ordered data are projected into this panoramic view of the galaxy and color-coded to display the temperature of the emitting material 
ill-posed inverse or "backward" problems which are inherent to the nature of space-borne observations. In many cases, the algorithms for extracting primary physical parameters from these space observations can best be derived by coupling them with numerical models that provide accurate solutions to the "forward" problem. Iterating between the model solution of the forward problem and observations than yields estimates of the physical parameters of interest. For example, one generally measures the electromagnetic intensities or the spectral energies at different frequencies in the electromagnetic spectrum which are not the primary parameters but rather integral functions that depend on these parameters through kernel functions such as absorption and emission properties of the intervening environment of the earth's atmosphere or molecular clouds surrounding stars and galaxies. Extracting the primary variables such as temperatures and moisture involves imposing certain physical assumptions or constraints on the nature of the solution. Four-dimensional satellite data assimilation techniques for incorporating derived or inferred physical parameters directly into dynamic models has proven a powerful method for inferring physical parameters. However, as a result of the need to iterate between complex model integrations and algorithms designed to physically extract parameters, computing requirements have soared exponentially. While such techniques have shown marked success in producing highly accurate observational fields, the resources have sorely been lacking to implement them in day-to-day operational space data processing. In the following, several examples of inverse problems are discussed.

\section{A. Solid Earth Modeling}

A scientific understanding of the earth as a system must penetrate, as well as encompass, the "solid" earth. After all, long-term weather and climate patterns are the forces altering mountains and ocean basin shapes. The aperiodic geologic time reversal of the magnetic field, which redirects cosmic rays into earth's radiation belts, is related to the geodynamics of the outer core. What causes the earthquakes and volcanic eruptions, which kill more swiftly and surely than a greenhouse-induced global warming, is related to tectonic plate motions. Could a catastrophic volcanic eruption cause regional or global mass extinctions? Can astrophysicists resolve a debate about the internal structure of stars, nebulas, or the universe while geophysicists debate the structure of the earth upon which they stand? These are but some of the problems being addressed by solid earth modeling.

One particular challenge for scientific supercomputing posed by the solid earth sciences arises from the modern science of geomagnetism (founded by Gilbert in 1600) and consists in understanding the behavior of the very core of our planet. The "solid" earth actually consists of a solid inner core, a liquid outer core, a solid lower mantle, a partially molten asthenosphere, and lithospheric plates identified with the uppermost mantle and the brittle crust. The electrically conducting core produces the earth's magnetic field by a dynamo process and directly affects the mantle

${ }^{1}$ The results presented in this section are based on work performed and material provided by C. V. Voorhies, Laboratory for Terrestrial Physics, NASA/Goddard Space Flight Center. through the exertion of torques, heat flux, and perhaps chemical reactions across the core-mantle interface. The dynamo process involves the interaction between the existing magnetic field and the fluid motion of the outer coreapparently thermochemical convections caused by the crystallization of the inner core from the outer core melt. The pattern of motions just below the core-mantle interface produces slow (or secular) variations of the geomagnetic field. These secular changes induced by the motions were measured by the Magnetic Field Satellite (Magsat) in 1980. However, these secular variables are better determined by including previous surface magnetic observations along with the Magsat data and, hopefully, through continuing satellite monitoring.

The 150-year-old problem of modeling the evolving, broad-scale geomagnetic field has now been generalized to include satellite data. However, deriving reliable error bounds on the resulting generalized Gauss coefficients apparently requires inverting data matrices of order $10^{5} \times$ $10^{5}$. Such error bounds are needed to test rigorously hypotheses about the structure and dynamics of the earth. The inversion of such huge matrices provides a major challenge for scientific supercomputing.

In the interim, combinations of data acquired by satellite magnetic mapping, surface and aeromagnetic surveys, and surface magnetic observatories have been used to derive low-order spherical harmonic models of the geomagnetic field. These data have been averaged to produce definitive geomagnetic reference field (DGRF) models at 5-year intervals, covering the period 1945 to 1980 . The models, which represent the evolving, broad-scale core fields rather than crustal magnetic anomalies, form eight sets of observations for which 120 Gauss coefficients are calculated from the fit of a spherical harmonic expansion of degree and order 10.

The Core Flow Model: Under assumptions appropriate for decade time changes and megameter length scales of interest for studies of the recent secular variations (sourcefree mantle/frozen-flux core), estimates of a steady fluid velocity at the top of the earth's core, which account for most of the geomagnetic secular variation, have been derived by Voorhies [1]-[3]. Assuming magnetic lines of force are frozen to the fluid core, motions of the fluid at the top of the core advect the two foot points of each magnetic field line threading the core-mantle boundary. The motion of the fluid, hence the field line foot points, induce continual rearrangement of the magnetic field lines outside the core. This causes the secular variations observed by the satellite and by the surface magnetic surveys.

In the source-free mantle approximation, spherical harmonic (DGRF) models of the geomagnetic field observed near the earth's surface, $B(a, t)$, where $a$ is the radius of the earth and $t$ represents a time-averaged epoch over 5 years, can be extrapolated through the mantle and used to map the broad-scale part of evolving radial magnetic flux density at the top of the core $B_{r}(b, t), b$ being the core radius and $t$ the same time interval as before. In the frozen-flux core approximation, the time evolution of $B_{r}(b, t)$ is related to the radial magnetic flux density field and the fluid velocity through a simple magnetic induction equation [4]. This leads to an undetermined inverse problem for the fluid motion at the top of the core because there is but one equation for the three fluid velocity components (two components of the surficial fluid velocity and one for the surficial conver- 
gence). The hypothesis of piecewise steady flow is used to overdetermine the inverse problem by admitting evaluation of the radial induction equation at many different magnetic field epochs without increasing the number of unknowns [5].

A weighted, constrained, damped iterative least-squares method for solving this inverse problem was developed [6] and applied to the eight DGRF models. This computational strategy involves defining and minimizing an objective function composed of a weighted residual variance (which measures how poorly the evolving DGRF models have been fit by the steady induction motion of field line foot points), an optional constraint requiring the core flow to be geostrophic, and a damping of small-scale flow structure. Each time-dependent element of the derived normal equation matrix is an integral over the core mantle boundary evaluated numerically on a $2^{\circ} \times 2^{\circ}$ grid. The result of each inversion is a "flow" estimate of the fluid velocity field at the top of the earth's core. Each flow predicts a magnetic field at the core-mantle boundary and a new set of elements for the normal equations. Thus the nonlinear inverse problem is solved iteratively. The nonlinear aspects of the problem appear initially mild, but increase dramatically within the duration of the interval over which the 35-year steady flow is presumed.

The weight matrices used were derived from the error covariance matrices for the 1945-1960 DGRF models provided by Langel et al. [7]. Error covariance and weight matrices were derived from the candidate models whose averages define the DGRFs at epochs 1965-1975. The 1980 (Magsat) model is taken as the initial epoch and considered perfect compared with the other DGRF models. Two kinds of weights are being explored. For the radial field weights, a scalar weight function was derived and each weight matrix element was determined from it by numerical integration. As expected, the weight function is heavy over areas of high data density (such as Europe) and light over regions of low data density (such as the southeastern Pacific Ocean and parts of the Indian Ocean). These weights allow an assessment of the information contained in the evolution of the broad-scale radial magnetic flux density at the earth's surface $B_{r}(a, t)$ called for by the DGRF models.

As a function of time, $\Delta B_{r}(a, t)$ in Table 1 shows the root
TABLE 1 Select Properties of DGRF Models and Uncertainty Estimates Based on Radial Field Weights

\begin{tabular}{cccc}
\hline \hline & & & $\frac{\Delta B_{r}}{t_{n}}$ \\
\hline 1980 & $\Delta B_{r}(t)$ & $\sigma^{\prime} B_{r}(t)$ & - \\
1975 & 0.0 & 0.0 & 5.47 \\
1970 & 318.9 & 58.30 & 12.91 \\
1965 & 612.7 & 47.47 & 19.79 \\
1960 & 899.2 & 45.42 & 45.08 \\
1955 & 1157.3 & 25.67 & 21.67 \\
1950 & 1427.2 & 65.87 & 13.19 \\
1945 & 1674.9 & 127.01 & 11.99 \\
\hline
\end{tabular}

mean square uncertainty of $B_{r}(a, t)$ averaged over the earth's surface. $\sigma^{\prime} B_{r}(a, t)$ gives the rms change in $B_{r}(a, t)$ relative to 1980 called for by the DGRF models, and the third column shows their ratios. The typical change in $B_{r}$ between 1945 and 1980 is 1929 nanotesla (nT). A typical rate of change in $B_{r}$ is about $55 \mathrm{nT} / \mathrm{yr}$. The estimated uncertainty in the largescale field is typically $51 \mathrm{nT}$ for the 1965-1975 DGRF models, less for 1960 , but more before then. The changes in the ratio $\Delta B / \sigma^{\prime} B_{r}$ reflect the competition between a longer time for secular change to accumulate and the deteriorating data coverage as one goes back in time.

Many calculations have been performed using the CYBER 205 in an attempt to find a steady fluid velocity field at the core surface which, starting at 1980 , can be used to advect field line foot points back in time and reproduce the DGRF models to within their estimated uncertainties. Fig. 4 shows a composite map of a fluid velocity field (arrows) and the downwelling (color) at the top of the core that was derived using the source-free mantle/frozen-flux core method outlined above, as well as the DGRF models from 1980 to 1945. Color bar units are $10^{-3} / \mathrm{yr}$, cool colors denote downwelling, warm colors indicate upwelling. A vector length of $10^{\circ}$ corresponds to $31.60 \mathrm{~km} / \mathrm{yr}$. This single steady flow accounts for $99.51 \%$ of the weighted variance in the evolution of the radial field called for by the DGRF models. The residual errors of $7 \%\left(0.49 \%^{-1 / 2}\right)$ are considered significant at the 2.96 standard uncertainty estimate. Such errors are expected in the source-free mantle/frozen-flux core approximation. They might also be due to spectrally incomplete initial conditions or time-dependent flow. The significance of the

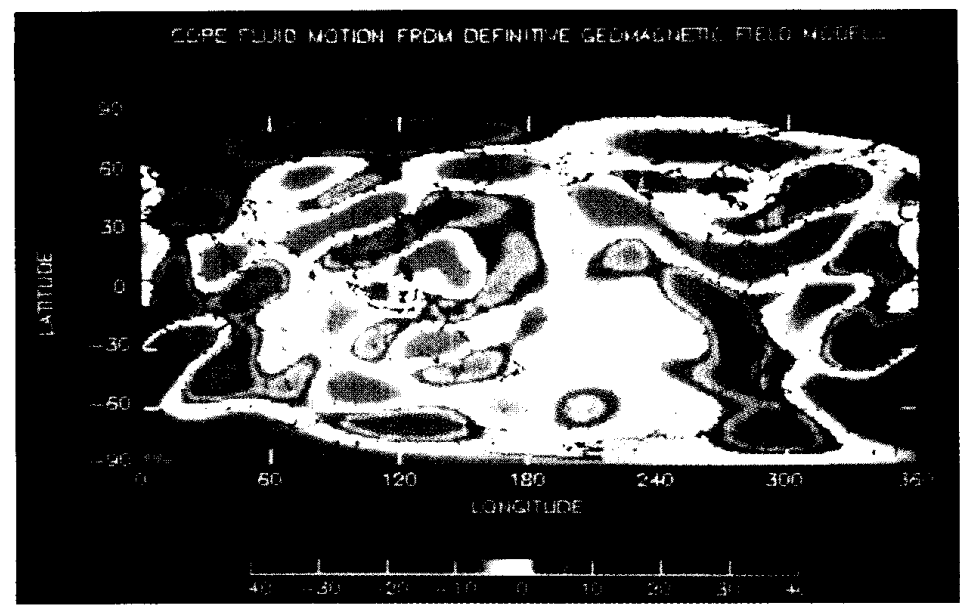

Fig. 4. Core fluid motion from definitive geomagnetic field models. Red areas show downwelling, blue areas upwelling. 
residual errors increases when the geostrophic flow constraint is imposed, indicating that steady and geostrophic flow hypotheses are incompatible.

The DRGF models and the geomagnetic data used to derive them provide an extremely powerful tool for probing the earth's deep interior. A weighted, constrained, damped iterative least-squares approach to estimating the steady fluid velocity field at the top of the earth's core can account for secular geomagnetic changes. The working hypothesis of steady flow appears reasonable for the simple source-free mantle/frozen flux core approximation. To improve the uncertainty estimates accounting for secular changes will require a more sophisticated earth model (which is already available), more reliable error estimates, and longer data records. The former is being developed and coded on a CYBER 205, but will require inversions beyond the capability of existing supercomputers. The latter rests on NASA supporting a long-term space program of magnetic field measurements.

\section{B. Earth Systems Modeling ${ }^{2}$}

Are the record-breaking heat waves experienced during the 1980s a signal of the arrival of the greenhouse effect caused by humans, as some scientists are saying? Will Antarctica's ozone hole continue to deepen annually? Is the dream of many modelers to someday predict interannual climate changes, such as the El Niño southern oscillation (ENSO), now being realized?

The recent recognition of the human ability to effect global changes in the environment requires that global processes be studied as a system, rather than through the traditionally distinct disciplines, so that interactions among land, ocean, and atmosphere can be better understood. To support these multidisciplinary studies, global observing systems are needed to provide the information necessary to investigate the interaction of earth processes through conceptual and numerical models.

A major limitation to answering these questions is our incomplete knowledge of the natural variability of the earth system on interannual and long-range time scales. Beginning in the mid 1990s, NASA, in collaboration with nations and scientists around the world, will put into space an earth observing system (EOS) to begin observing the global variability of many of these processes, which naturally occur on time scales of up to 10 years. In addition, an interdisciplinary research program is being initiated this year to assess the impact on climatic processes of external influences and those of humans.

Understanding such global phenomena involves both an analysis of the wealth of data that EOS and other observing systems will provide over the next decades, and the development of a theoretical framework around which to organize these observations. Because of the complexity of the earth system, detailed numerical models have become standard tools in this endeavor. In most of the earth sciences, numerical models are currently being used to analyze data and to develop and test theories.

To understand the large-scale long-term variability of the

${ }^{2}$ The results presented in this section are based on material provided by P. Schopf, Laboratory for Oceans, NASA/Goddard Space Flight Center, and reflect work performed by P. Schopf, Y. Sud, C. Parkinson, R. Koster, and M. Suarez. earth system requires studying many natural phenomena. We choose to illustrate here a few earth science problems that have recently been addressed by modeling.

1) How do the ocean and atmosphere interact to cause long-term variability, such as the El Niño southern oscillation phenomenon?

2) What causes the interannual variability in the distribution and the volume of sea ice and how does that variability affect the climate?

3) How do variations in ocean circulation and incident radiation affect biologically controlled levels of $\mathrm{CO}_{2}$ and photochemical reactions at the ocean surface?

4) How do variations in soil moisture and vegetation affect atmospheric dynamics, regional climate, and chemical fluxes from the surface to the atmosphere?

The current state of earth science modeling and computer technology has reached a capability that appears to make possible a comprehensive approach to the study of the earth. The first attempts to explore interactions between the ocean and the atmosphere, between atmospheric dynamics and chemistry, between ocean biology and ocean circulation, and between vegetation and the hydrological cycle have met with reasonable success. In this section we briefly describe some illustrative models of uncoupled system components and examine their realism by comparing the simulations with observational data.

Ocean Circulation Model: Although there are various ocean models being used within the oceanographic research community, we describe the ocean model [8] that is a quasi-isopycnal (constant-density) coordinate model with many layers representing the surface mixed layer, the upper thermocline (a region that interacts directly with the mixed layer), the main thermocline, and the abyssal ocean. In addition to the vertical coordinate, which is arranged to minimize artificial cross-isopycnal mixing, the model has full nonlinear dynamics, very low horizontal diffusion, and Richardson number dependent vertical mixing [9]. To date, the model has been run most extensively in the fast reducedgravity mode, in which horizontal pressure gradients in the abyssal ocean are assumed to vanish. The equations of the model are formulated in terms of stream functions and vorticity, making use of the barotropic assumption. However, we expect to reformulate the model to calculate the external gravity mode in the near future. For many of the problems to be addressed, particularly in the tropics and midlatitudes on short (less than 100-year) time scales, the use of the reduced-gravity formulations provides adequate representation of the near-surface variability-particularly for sea surface temperature (SST) and surface height. In the polar regions, the connection with the abyssal ocean is of great importance, and the external mode currents have a significant impact on surface flow, stability, and therefore, ice edge effects, SST distributions, and surface height in these regions. The model is also being used for near-realtime simulation of the tropical Pacific, as influenced by the assimilation of satellite altimetry.

Fig. 5(a) shows an instantaneous realization of the SST fields on November 15 of the 25 th year of a simulated integration with this model. The existence of short waves between $5^{\circ} \mathrm{S}$ and $5^{\circ} \mathrm{N}$ in the eastern Pacific in this model simulation compares favorably with those observed [10]. The model has identified their origin in barotropic instabilities occurring from the shear between the north equa- 


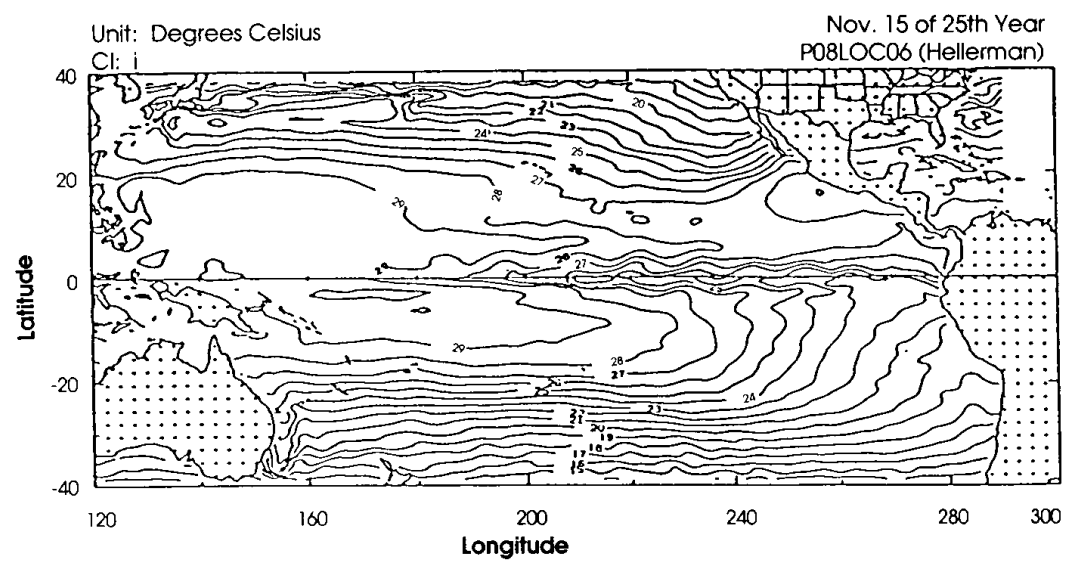

(a)

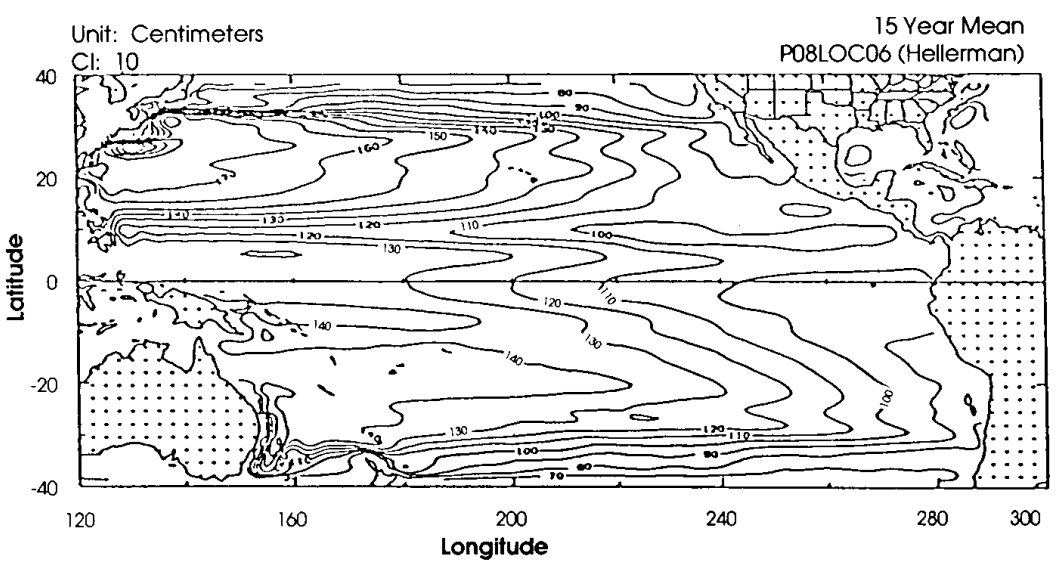

(b)

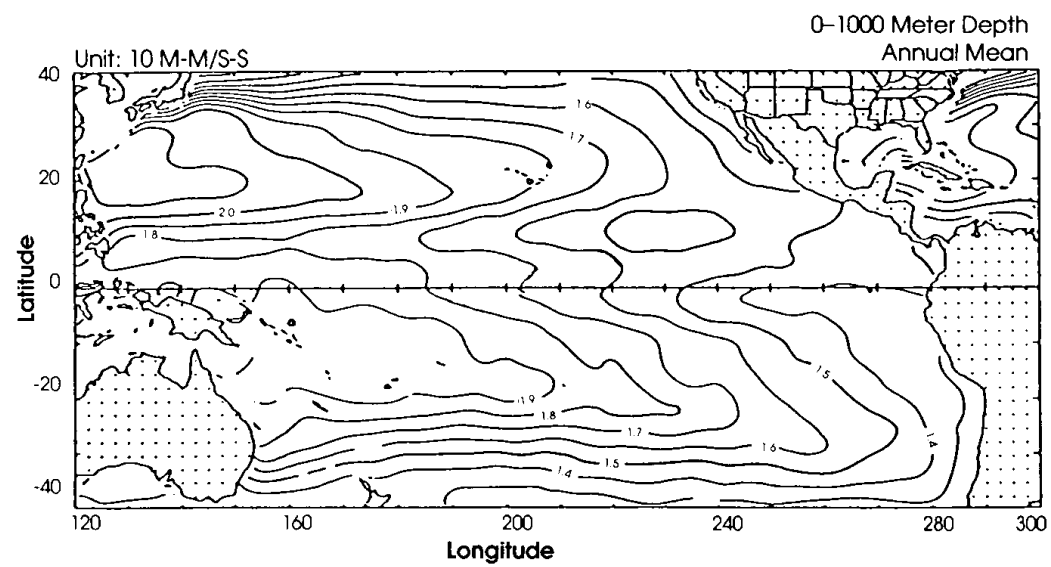

(c)

Fig. 5. Sea surface temperatures. (a) Model simulated on November 15, year 25. (b) Simulated annual mean surface height. (c) Observed dynamic topography (Levitus).

torial countercurrent (NECC) and the north equatorial current.

Fig. 5(b) and (c) shows simulated and observed surface height climatology [11], based on a 25-year run, forced with monthly wind climatology. The equatorial tilt of the surface height and surface height variations associated with the NECC are well simulated, as is the general character of the subtropical gyres. Differences between simulation and observations are comparable to differences between simulations with different wind climatologies. Details of the Kuroshio current and the effects occurring near the meridional boundaries are influenced by the limited domain used in this simulation.

Fig. 6 shows the depth structure of the model fields 

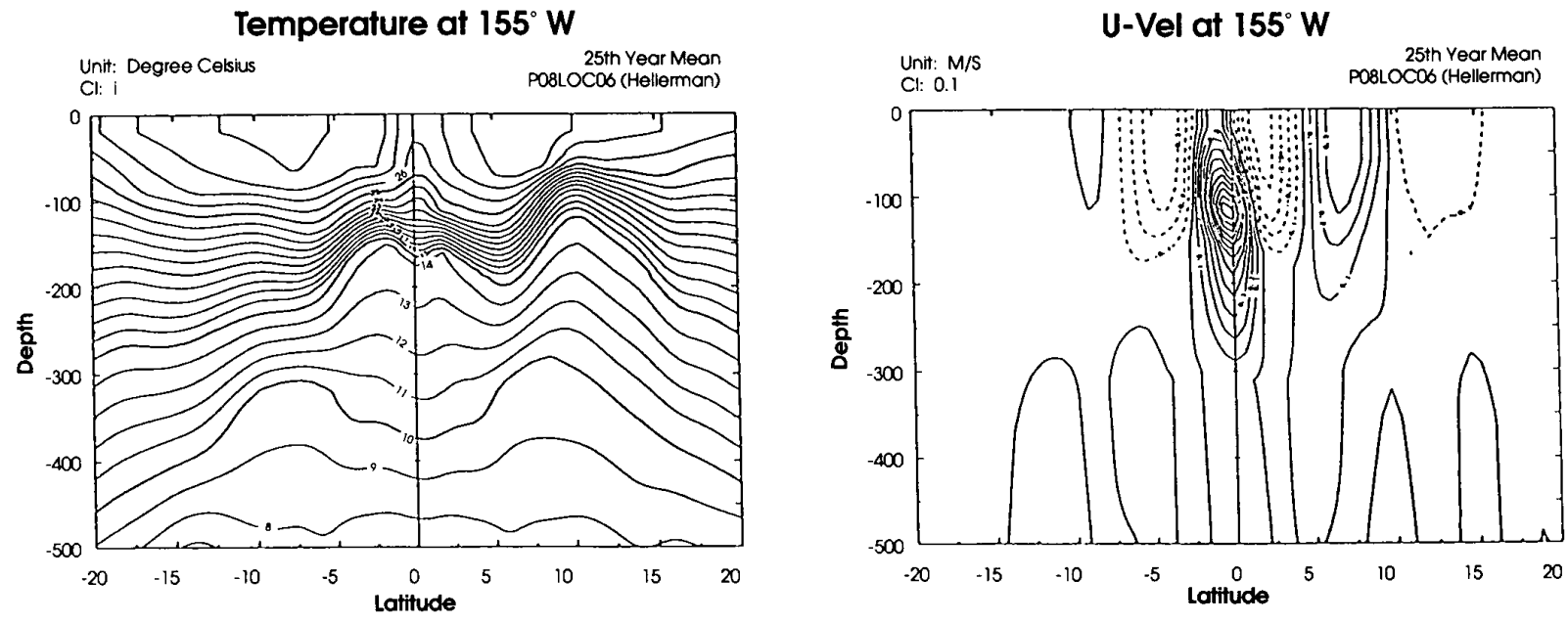

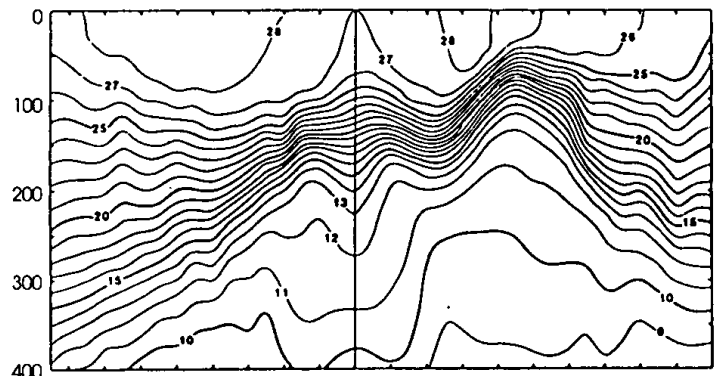

(a)

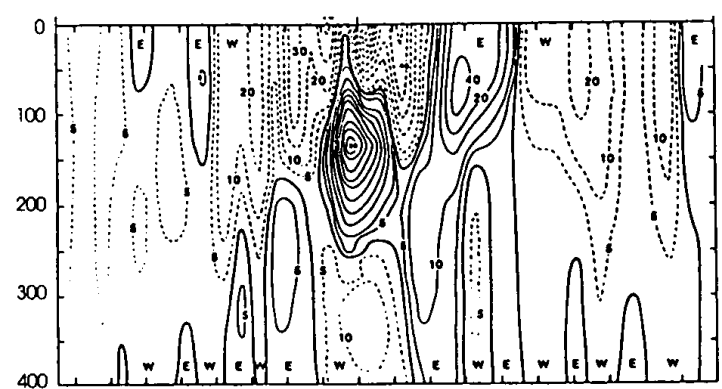

(b)

Fig. 6. Depth structure of model fields between $20^{\circ} \mathrm{S}$ and $20^{\circ} \mathrm{N}$ along $150^{\circ} \mathrm{W}$ as an annual mean.

between $20^{\circ} \mathrm{S}$ and $20^{\circ} \mathrm{N}$ along $150^{\circ} \mathrm{W}$ as an annual mean. For comparison, data from the Hawaii-Tahiti shuttle experiment are shown [12].

Fig. 7 shows the sea level at Fanning Island $\left(4^{\circ} \mathrm{N}, 159^{\circ} \mathrm{W}\right)$ as simulated over the last 5 years of the model run (thin solid lines) in comparison with the climatological data from the tide gage, shown with the dashed line [13].

Atmospheric Circulation Model: The atmospheric general circulation model was recently developed at Goddard's Laboratory for Atmospheres for long-term climate studies. It is an $\mathrm{N}$-level primitive equation model on a standard sigma (pressurelike) coordinate. Its prognostic variables are the two horizontal wind components, the potential temperature, the water-vapor mixing ratio, and the ozone mixing ratio.

The vertical discretization follows closely that proposed by Arakawa and Suarez [14]. It conserves total energy, potential temperature, and angular momentum. The horizontal discretization is based on finite differences on latitude-longitude coordinates, using a staggered $\mathrm{C}$ grid. The momentum advection scheme is enstrophy (that is, generalized vorticity) conserving, and the advection scheme for all scalars conserves the quantities and their squares. The latter are being modified for ozone and other trace quantities to be included in the future. An explicit leap-frog scheme is used for the time finite differencing. Linear stability is maintained at high latitudes by applying a selective

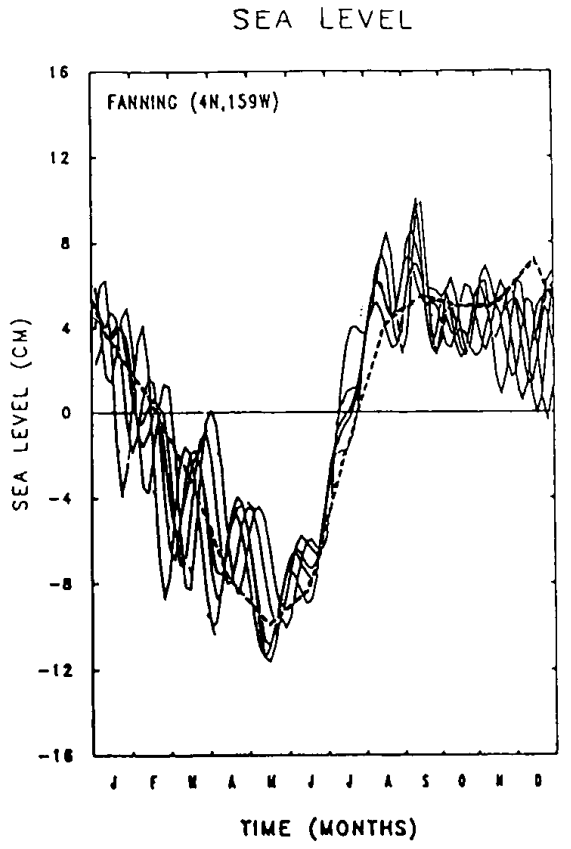

Fig. 7. Sea level at Fanning Island $\left(4^{\circ} \mathrm{N}, 159^{\circ} \mathrm{W}\right)$, simulated over the last 5 years of the model, as compared to climatological data from the tide gage. 
zonal smoothing to the pressure gradient and the mass fluxes near the pole. In addition a weak eighth-order Shapiro filter is applied to the temperature and wind fields to avoid nonlinear computational instability.

The model includes a full complement of physical parameterizations. The infrared and solar radiation parameterizations follow closely those described by Harshvardhan et al. [15]. In the long wave, water vapor absorption is parameterized [16], as are the $15-\mu \mathrm{m}$ band of $\mathrm{CO}_{2}$ [17] and ozone absorption [18] with modifications [19]. The short wave follows the Davies parameters, as described by Harshvardhan et al. [15].
The moist convection parameterization was developed recently and is not yet published. It is based on the wellknown Arakawa and Schubert [20] parameterization, but solves an adjustment problem by considering simultaneous interaction among all possible cloud types. The new parameterization considers only one cloud at a time, and rather than adjusting fully every hour or two, it does a series of partial adjustments, tending to relax the state to equilibrium, and is several times faster than the earlier Arakawa-Schubert parameterization.

The model uses various spatial resolutions, as shown in Fig. 8, depicting the winter and summer precipitation

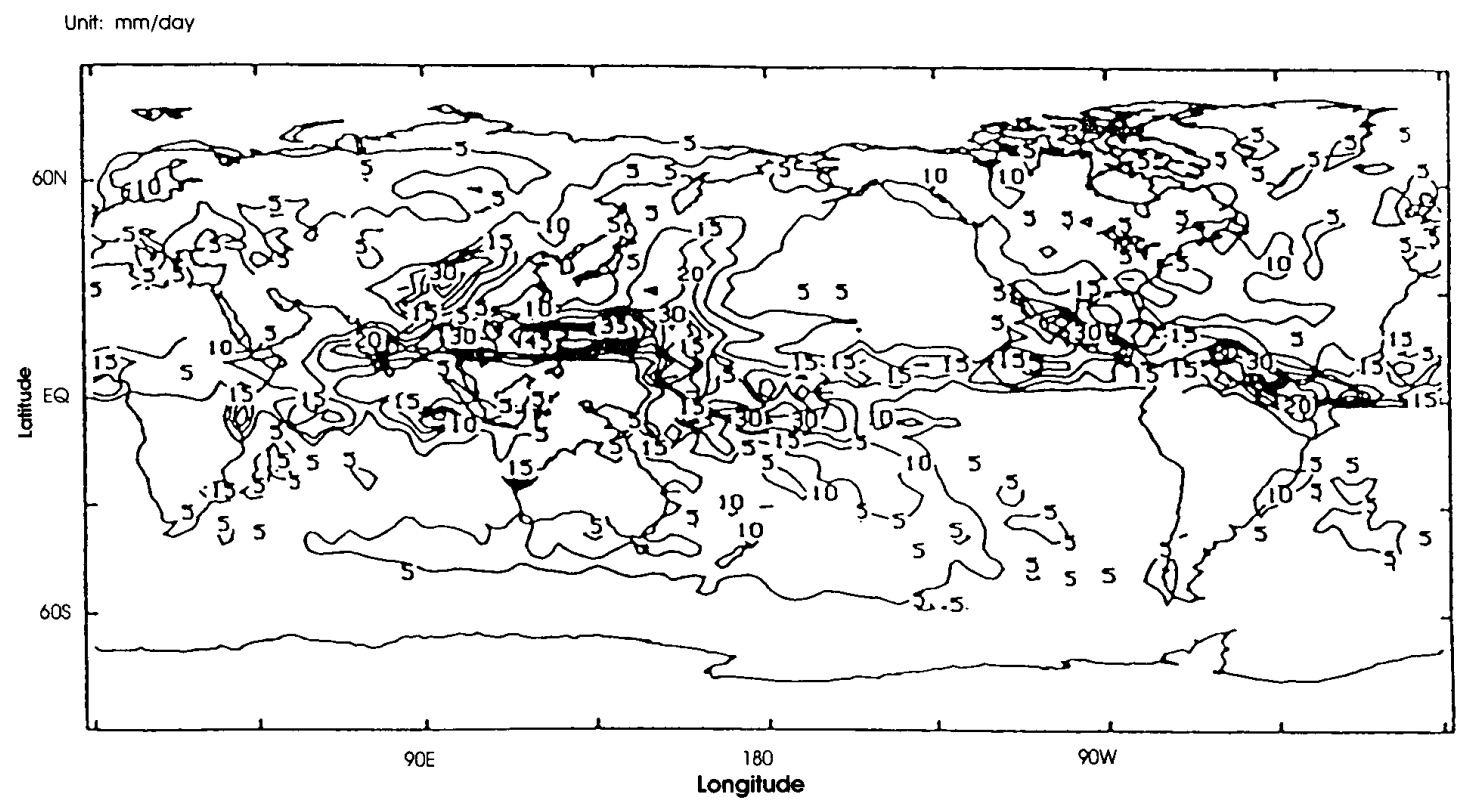

(a)

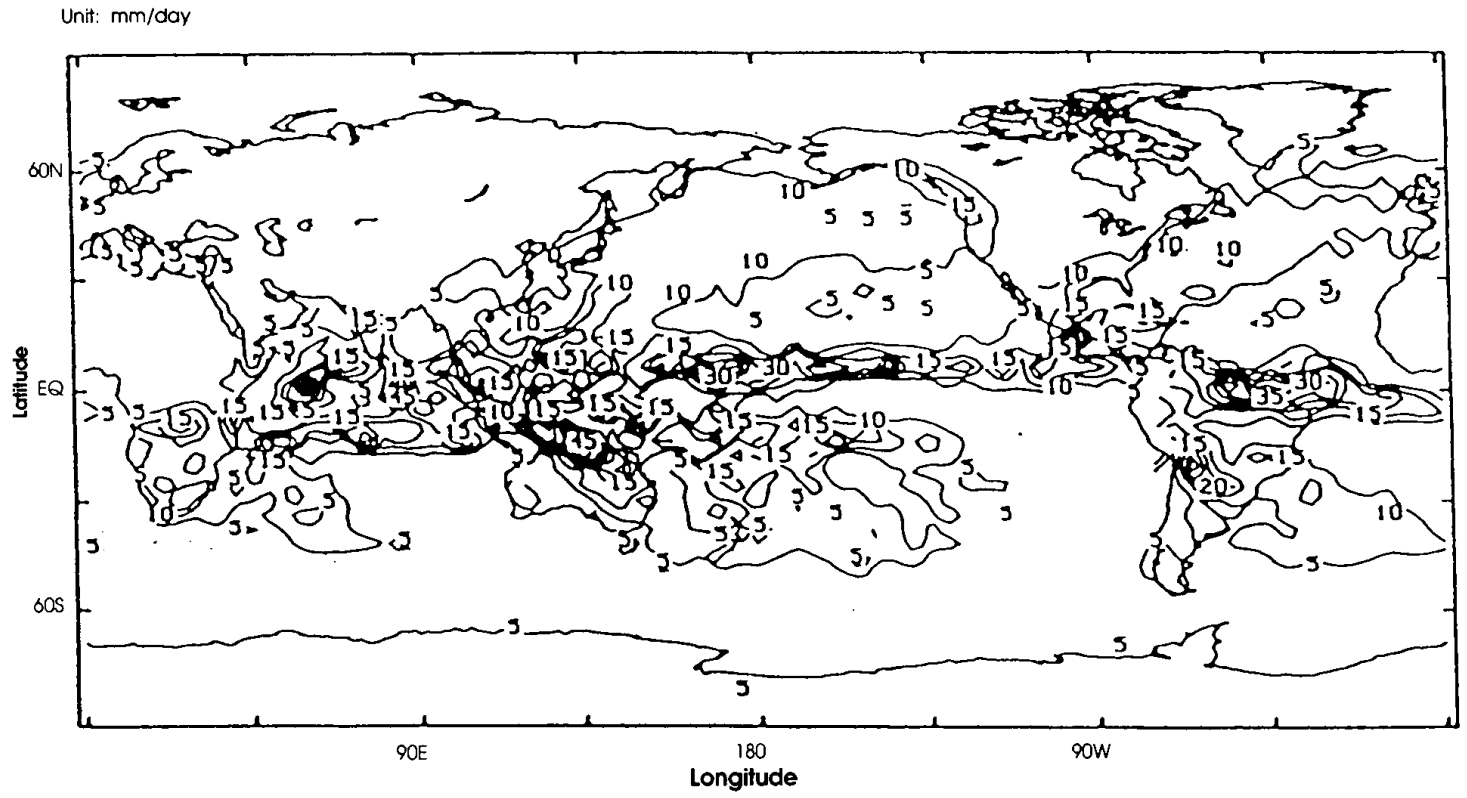

(b)

Fig. 8. Simulated total precipitation. (a) Summer. (b) Winter. Units are $\mathrm{mm} /$ day. 


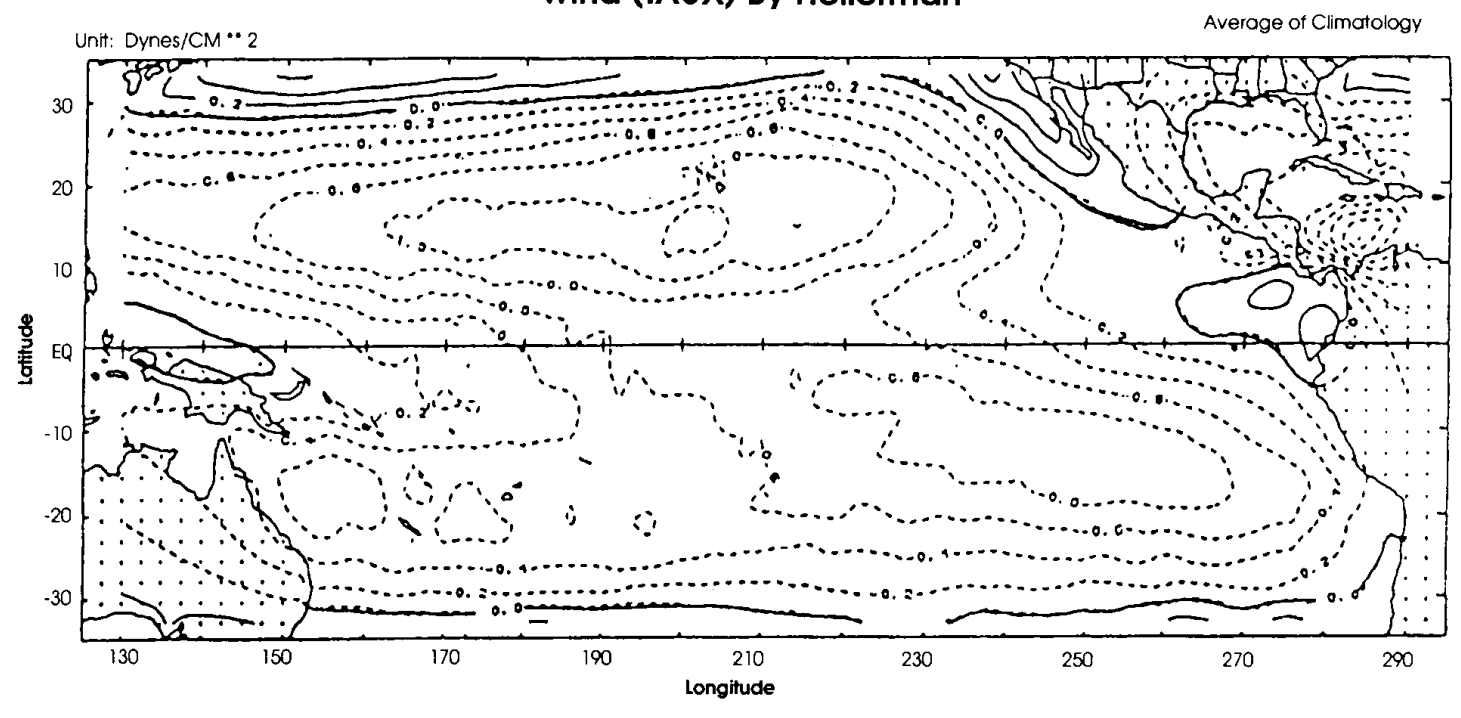

ZON Wind Stress (Annual)

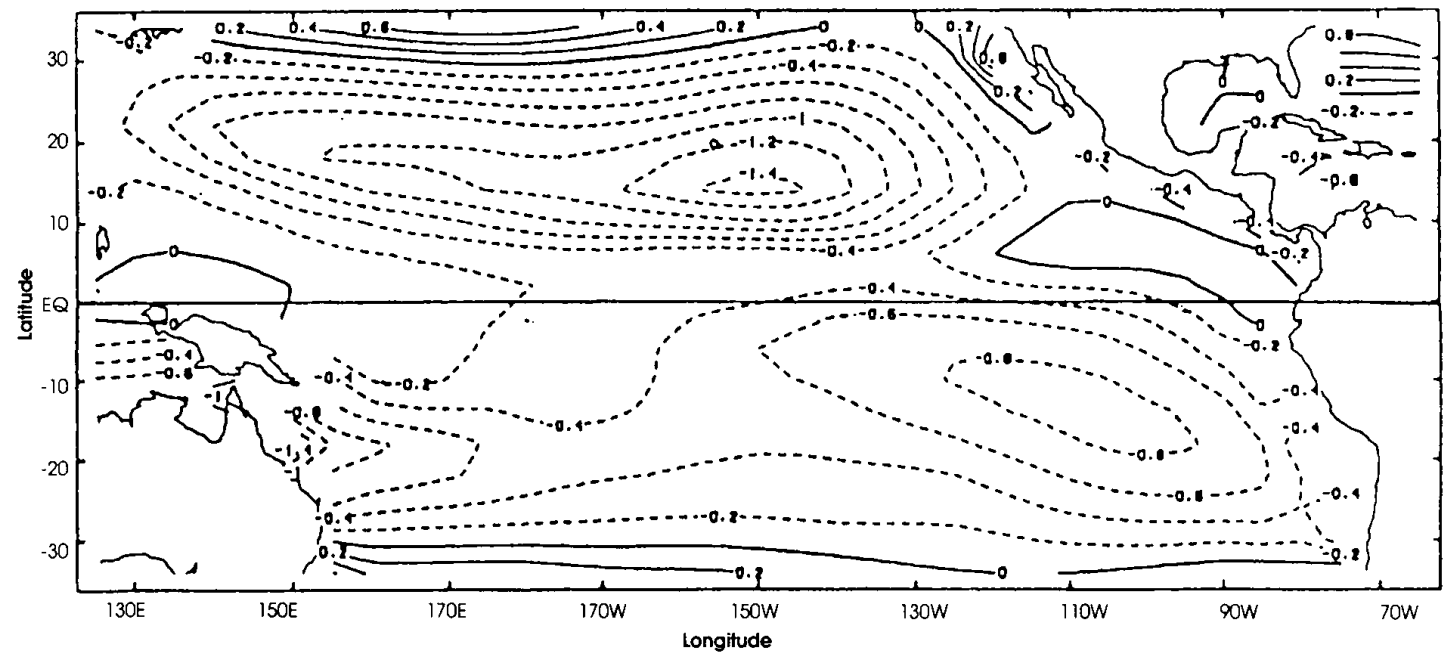

(a)

Fig. 9. Annual mean surface wind stress acting on the ocean. (a) Zonal (east-west) component. (b) Meridional (north-south) component. Upper panels are "observed" stresses estimated by Hellerman; lower panels are stresses simulated using ARIES, a climate GCM developed at GSFC. Units are dyn $/ \mathrm{cm}^{2}$.

obtained from the $4^{\circ} \times 5^{\circ}$ version with eight levels. This version simulates a day in 25 seconds of CYBER 205 time; with a diurnal cycle the same calculation takes 35 seconds. Fig. 9 compares observed annual mean wind stresses as estimated by Hellerman with those simulated using the Goddard atmospheric circulation model.

Sea-lce Model: A dynamic-thermodynamic model of seaice distribution, thickness, and concentration based on a dynamic-thermodynamic model [21], with a revised formulation of ice dynamics incorporating nonlinear ice rheology [22], has been used to simulate the seasonal cycle of sea ice in both polar regions at a horizontal resolution of approximately $200 \mathrm{~km}$ with an 8-hour time step. Ice thermodynamics are calculated through energy balances at the interfaces between air, snow, ice, and water, with the following energy fluxes included: incident and reflected solar radiation, incoming and outgoing long-wave radiation, sensible and latent heats, conduction through the ice and snow, an ocean heat flux, and the fluxes from the melting of the ice and the freezing of the water. Ice dynamics are calculated through a momentum equation balancing air stress, water stress, dynamic topography, and Coriolis force, with a subsequent adjustment for internal ice resistance. The model has been used in several applications, specifically in simulating the effect on the ice cover of anticipated atmospheric warming and in simulating the impact of winds on a larger open-water region in the midst of the Antarctic sea ice. 


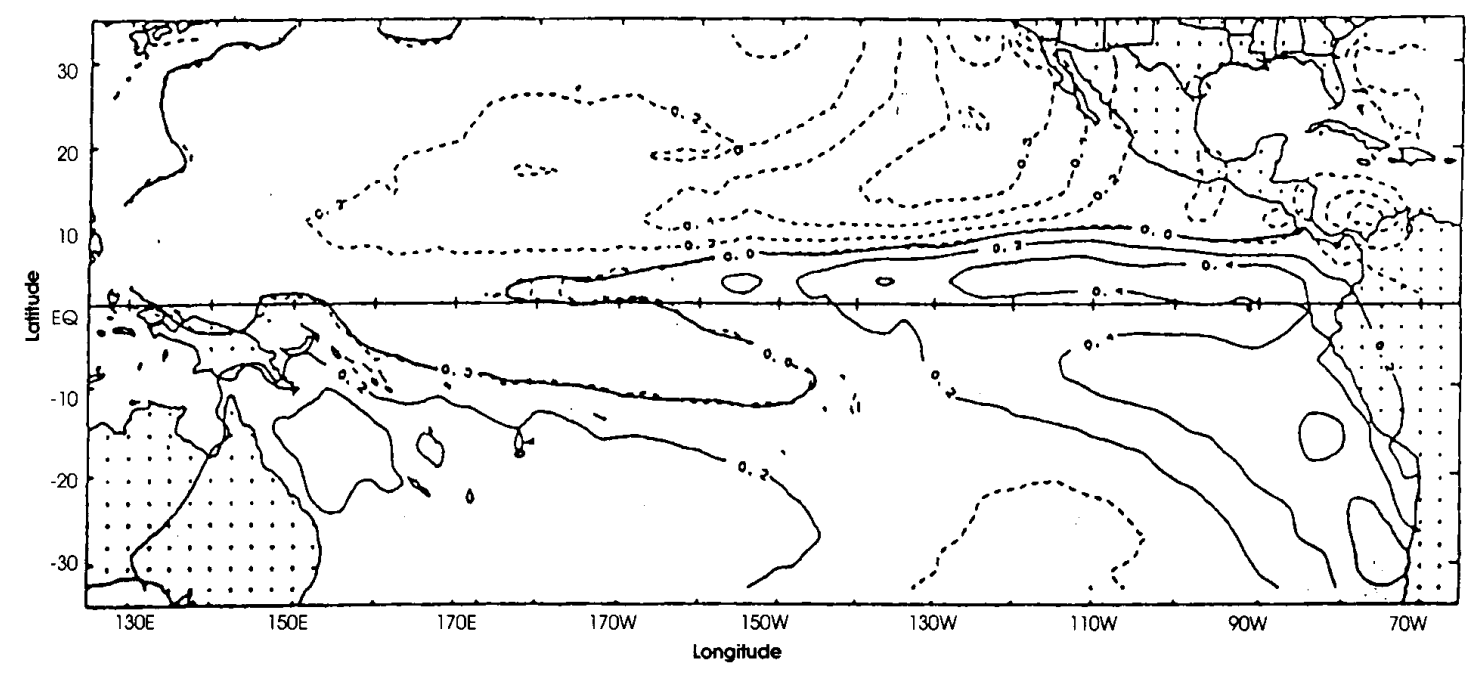

MER Wind Stress (Annual)

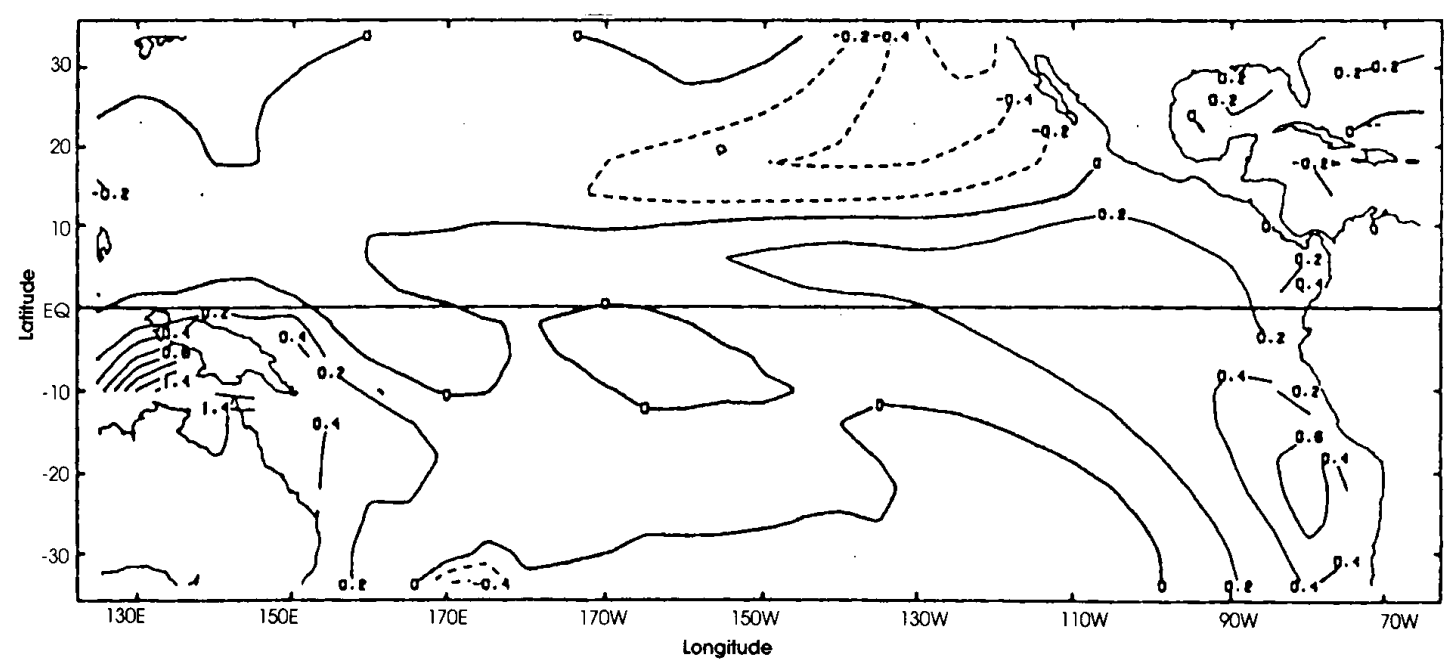

(b)

Fig. 9. (Continued)

The nonlinear ice rheology [23] allows internal ice resistance to be included directly as a term in the momentum equation. The constitutive law of sea ice is constructed on the basis of the Reiner-Rivlin theory and determines isotropic stress based on ice concentration and thickness, and shear viscosity based on ice concentration, thickness, and strain rate. Moreover, the isotropic stress exists only when the ice is converging.

In recent work simulating coupled ice-ocean processes, Hakkinen and Cavalieri [22], [23] demonstrated the importance of ice-induced mixing. In the presence of cooling, mixing is enhanced and may lead to deep convection. Fig. 10 displays results from this model, showing the evolution of the ice concentration, mixed-layer warming, and mixedlayer salinization.
Land Surface Processes Model: A simple biosphere (SiB) model is based on attempts to model the vegetation's active control over the surface heat and moisture budget [24]. The land surface is represented as a vegetation canopy over two soil layers-the upper soil layer and the encompassing root zone. The model has only four prognostic variables, the ground temperature (assumed equal to the canopy temperature), the water contents of the two soil layers, and the water content of the canopy interception reservoir.

The parameterization of evaporation is based on the Penman-Montieth formulation. The resistance network formulation is much simpler than that of $\mathrm{SiB}$. The vegetation and aerodynamic resistances are assumed to act in series, with the canopy idealized as a single leaf. Canopy resistances are computed as functions of the soil moisture con- 


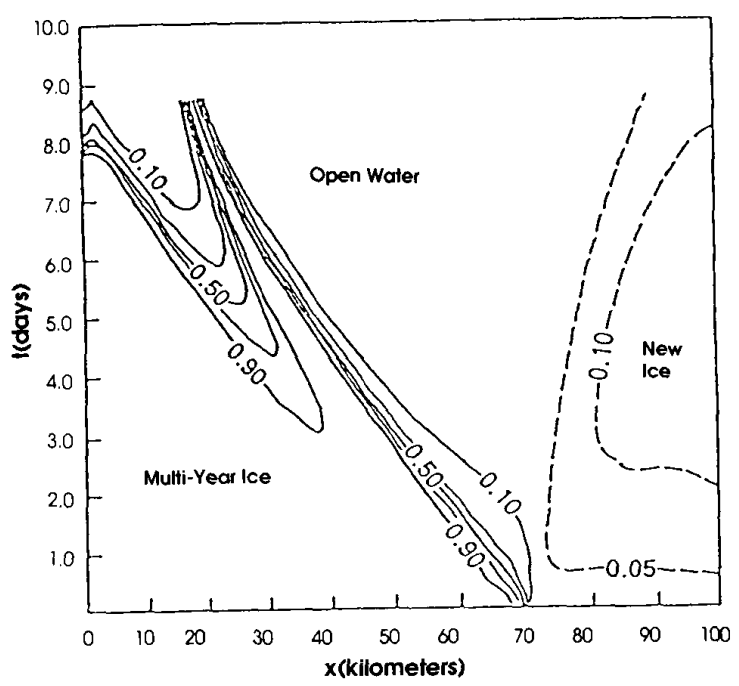

(a)

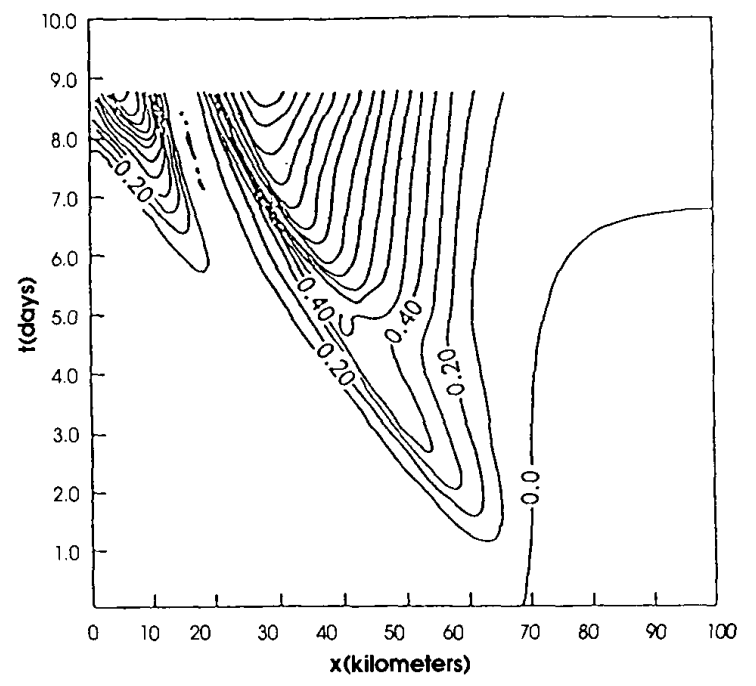

(b)

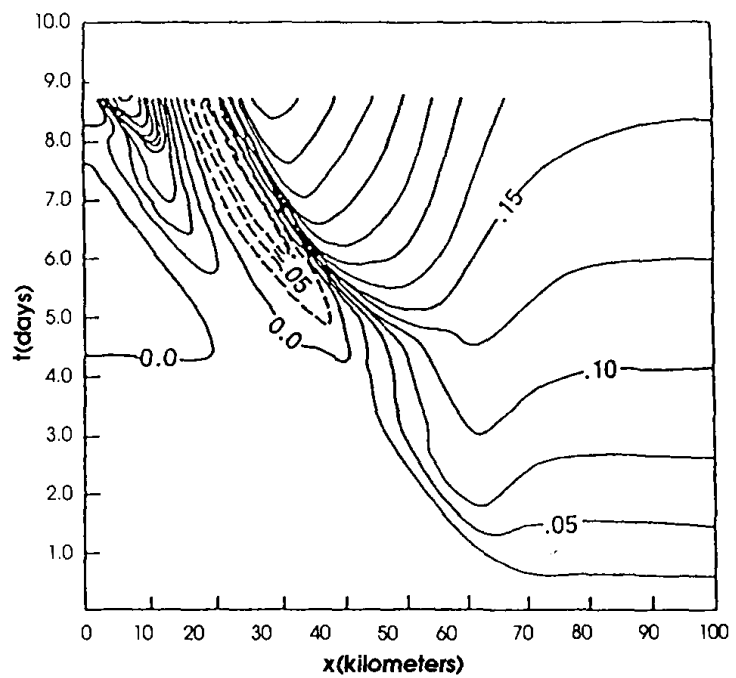

(c)

Fig. 10. Evolution of (a) ice concentration, (b) mixed warming, and (c) mixed-layer salinization. Units are ${ }^{\circ} \mathrm{C}$.

tent, the solar radiation flux, the water vapor deficit in the surrounding air, and the atmospheric temperature.

A statistically determined fraction of the precipitable water falling on the canopy is assigned to remain on the vegetation leaves rather than be absorbed by the soil. Evaporation from this canopy interception reservoir is computed assuming no canopy resistance. The atmosphere will always exhaust this reservoir before drawing moisture through the plants.

The first version of the model allows only one vegetation type per grid square. We assume that neighboring types act independently on short time scales (minutes) and interact with one another at longer times through the modification of the grid scale quantities.

In Fig. 11 precipitation results averaged over four simulation experiments for the month of July for the years 1979 , 1980, 1981, and 1982 [Fig. 11(a)] are compared with observed precipitation estimates from satellite data [Fig. 11(b)]. Fig. 12 shows the precipitation differences with and without a prognostic biosphere. The areas of red indicate that the biosphere model has reduced the precipitation in most regions where the NO biosphere simulation exceeds the observations. With the biosphere, global rainfall goes down by $0.55 \mathrm{~mm} /$ day and over most land regions of the northern hemisphere (Eurasia, Africa, and the Americas), in much better agreement with the observations shown in Fig. 11(b).

This type of integration with the general circulation model (GCM) is only possible with modern high-speed and largememory computers. To do an even better job, we need machines with larger memory and greater speed so that we can run global experiments with 40 times more resolution $(10 \mathrm{~km}$ instead of $400 \mathrm{~km})$, which implies bigger and faster supercomputers. This will provide the kind of rainfall structure that we see in observations. 


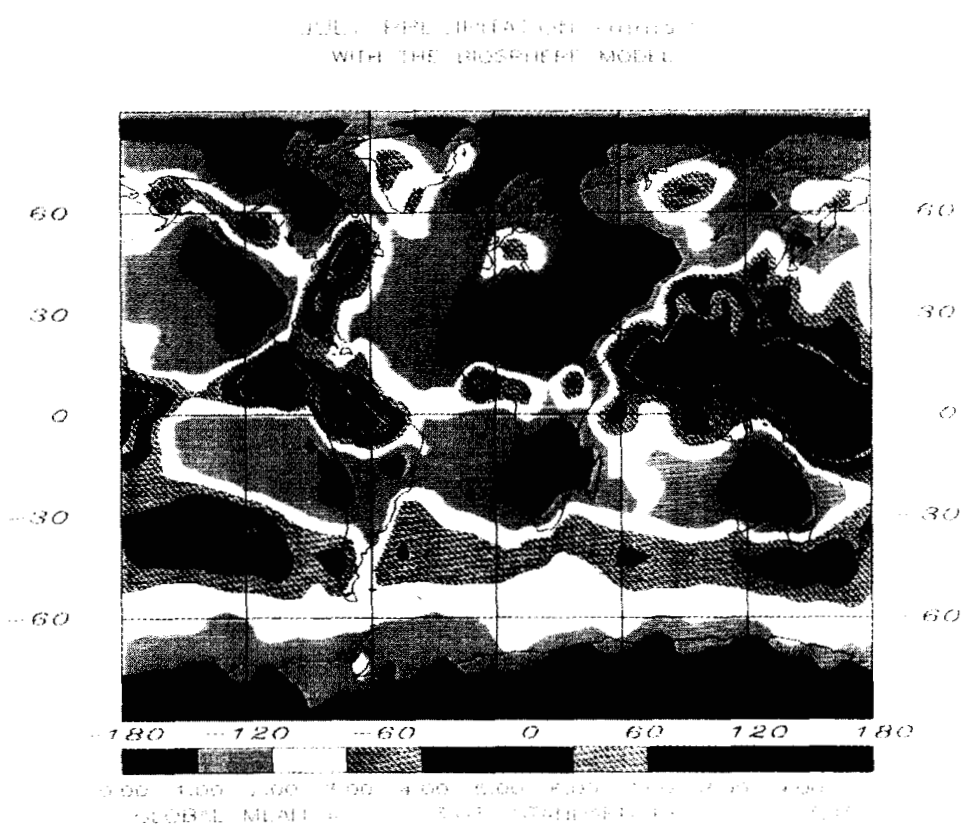

(a)

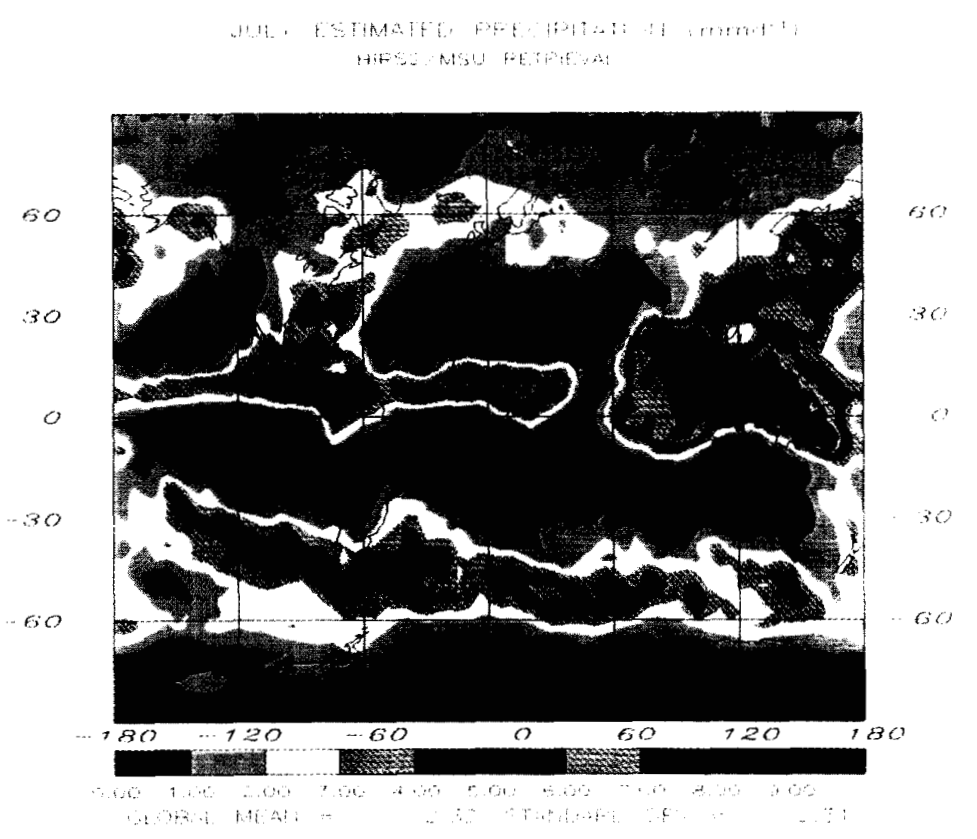

(b)

Fig. 11. Modeled precipitation results averaged over four simulation experiments for July 1979, 1980, 1981, and 1982 (a) are compared with observed precipitation estimates from satellite data (b). Improvements over the corresponding simulations without the biosphere can be seen. Global rainfall goes down by $0.55 \mathrm{~mm} /$ day and over most land regions of the northern hemisphere (Eurasia, Africa, and the Americas). The rainfall is reduced very significantly because the biosphere changes the Bowen ratio. 




Fig. 12. Preciptation diferences, wh and whou an actue biospher.

\section{Parallel Processing Applications of Farh Remote Sensing ${ }^{3}$}

Satellite observing systems are capable foday of snecinally imaging the earth's surface and amosphere at data rates far exceeding our ablity lo processor extrac their full information content in any practical manner. This has resuled in limited duy ycle space use ofinstruments hry ing high data rates and, very often, archiving vast amounts of unprocessed data for possible future access. With advances in charge coupled device $(C \mathrm{CD}$ technology, more of the passive sensors with lower data rates are cvolving into imaging sensors. As we move into the permanentiy manned space station era, an earh observing system is planned to provide coninuous mages with such insmemenis that will monitor the long term changes in the earth's biophysical and chemical cycles. Along with developments in passive sensing, massive image data sef are also being produced by space borne active sensors that use verylarge real or synthetic aperiures. Magellan, to be latuched in 1989, will attempt to image the surdace of Venus wh an active adar system that can penetrate the dense pemanen cloud cover of that planet. The Sovet Marner mission plans so place a satellte around Phobos, the outer moon of Mars. to produce a threedimensional map of its entire surbe. A solar oscillating image bult by NASA to lly on the Euro. pean Space Agency $50 H O$ mission plans to mage continwously the surface of the sun in order to inter the deep interior thermal structure of the sun. The impact of these observing systems could lead to a revolution in our understanding of the earth and space systems, but to achieve that goal, radically new technologies based on parallel com.

The restls presented in this sectom are based on wok per formed and materlal providsd by H Ramapriyan, I. Strong, Thton, 1. Dorband, Jischer, and M. Halem ot the Spare Data and Compating Oivibun, NASACoddard Space Hight Center. puting architectures and agorithms wil have to be devel. oped.

Recognizing the enomous image processing requirements for the earhwscience sensors and similar inaging requitements emerging in the planetary solar-terrestrialand astrophysical sciences, NASA embarked on a pilot project to buld massively parallel processor that can begin addreseng these probiems [25]-[27]. The massively paralle processor (MPP), delivered in 1983 by the Coodyear Aer. ospace Corporation and made awallable for research to the general NASA science community, has led to some interesting supercomputing applications. Two examples from this research are presented.

Topographic Modeling from Space: In this section, we presen resulis of a fully automated stereo matching algorithm daveloped for the MPP to determine terminelevalion datia from spacebone synthetic aperture radar (SAR) imagery data.

The algorihm, which is based on a lowlevel vision tech nique [28], [29], was applied 10 a pair of overlapping syr. thetic aperturs radar mages acquited during the october 1984 Space Shutte Challenger ilght. The images were taken over a plateau region of Bangladesh at incidence angles of $25^{\circ}$ and $42^{\circ}$, respectively. As a result of the viewing geom. etry, a linear scale change in the slant range istereopsis drection is first applied to the second itest image to pro duce the same pixel resolutonas the first lreferencelimage. For each pixel in the reterence image, a rectangular neight bothood surounding if is correlated with a set of neigh. borhoods corresponding to each point in a search area in the test mage. The disparity that is, the amoun of translation on a pixelbypixel basis needed to register the images is then detemined by associating in the stereopsis direction the location of the corresponding center pixel of the neighborhood within the search area with the maximum correlation value. Badly matching regions of the dls- 


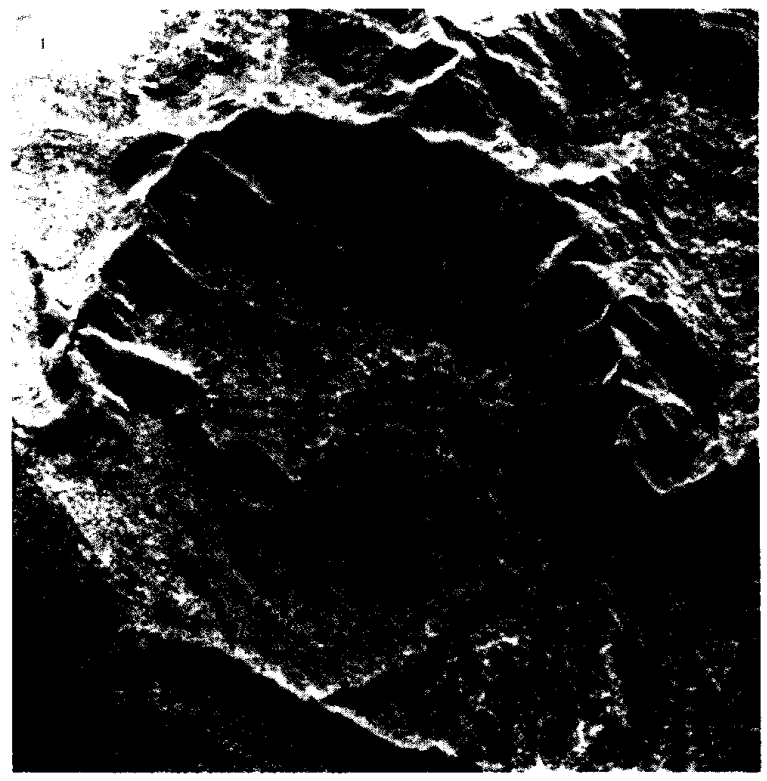

(a)

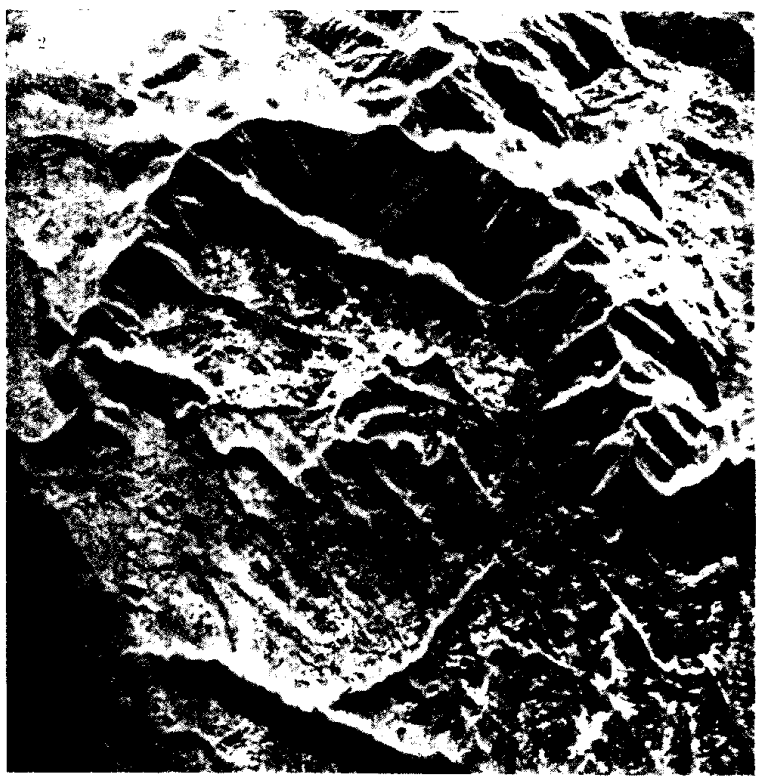

(b)

Fig. 13. (a) Pseudostereo overlay of SIR-B shuttle images. Red and green indicate max imum radar reflection values, respectively. (b) Overlay after application of two iterations of matching algorithm. The blending of red and green into yellow indicates good agree. ment resulting from warping

parity function show up as adjacent pixels with jumps greater than 1 and are filled in by an interpolation procedure based on an analog of a diffusion process applied to the region. The disparity function is then used as a geometric correction function to warp the test image so that it more closely matches the reference image. The algorithm is iterated for the entire image, with successively smaller neighborhoods. Fig. 13 shows the reference and test images overlayed after the initial linear warping and edge alignment. The two images are displayed with red and green, respectively, as maximum brightness values. One readily observes a broad plateau region with a surrounding river valley on the right. Fig. 13(b) shows the results of the matching algorithm after two iterations, starting with rectangular neighborhoods of 25 by 25 pixels and 13 by 13 pixels, respectively. The coincidence of the red and green in contrast with Fig. 13(a) indicates the agreement of the match. Disparity is linearly proportional to elevation, with dark areas corresponding to low elevations. By a three-dimensional graphics-rendering capability developed for the MPP [30]. Fig. 14 displays a three-dimensional view of this region illuminated from a source approximately at the location of the shuttle at $42^{\circ}$ from vertical. Overlayed on the disparity functions are the observed brightness values. An examination of a cartographic contour map of the same region available at NASA shows very good feature agreements between plateau, river, valleys, and height gradients for the entire region. This one imaging calculation required more than $10^{11}$ operations, in a little less than a minute, which could not have been computed in less than a week of dedicated, around-the-clock VAX $11 / 780 \mathrm{CPU}$ time. More importantly, if one is to attempt to map the surface of a moon such as Phobos, thousands of such images will need to be stereo mapped. These results establish the viability of generating reasonably accurate automated estimates of terrain height from space.

Motion Modeling from Time-Varying Imagery: Unlike elevation mapping by stereo matching, where surface features are stationary in time and all disparities in multi-incident viewing image pairs are attributed to variations in elevation, detection of motion is a related problem that assumes "identical viewing geometries" in pairs of images and attributes local differences to motion within the scene. In this study, we report on algorithms for the detection of ice motion developed for the MPP [31] and some preliminary results achieved thus far for two-dimensional ice motion detection.

The study is conducted with data acquired in 1978 from nearly identical viewing angles of the synthetic aperture radar sensor flown on Seasat. We assume that a 3-day interval as used in this study is sufficiently small that the dynamics of motion will not cause distortion of the ice surface to the extent that an area in one image will not be recognizable in the other.

A major difference to be considered in extending the ideas of the automated stereo matching algorithm is the need to account for rotation and the fact that large changes are possible between image pairs. Because of the latter condition, we first apply a global cross correlation between reference image and test image. This is performed efficiently by assuming periodicity in both horizontal and vertical directions and performing a two-dimensional fast Fourier transformation. Because translations of large coherent regions produce local maxima, or peaks, in the global cross correlation, the locations of these peaks can be used to define an approximate translation vector between the corresponding pixel of a given peak and the center pixel of the global correlation. After translating the test images by an 


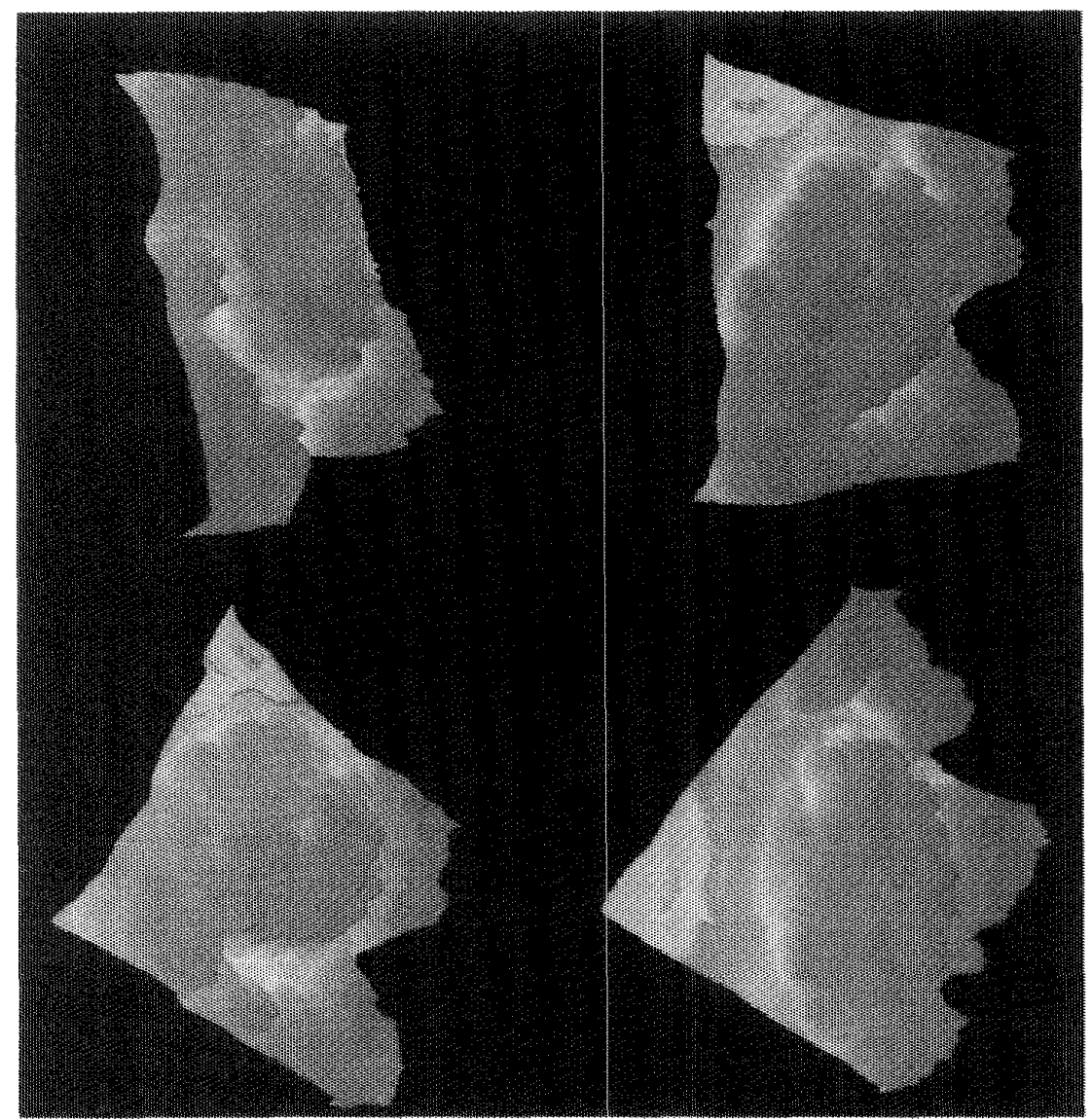

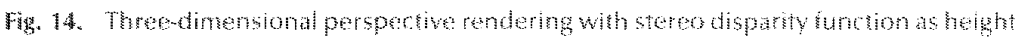

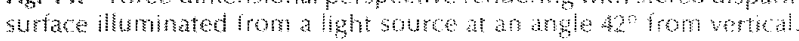

anoun comesponding lo a five local maxmum, the local correlation technique of the type used tor theo mathing is then used widenthy the corresponding regions of pont in he test mage which maththose in he tewencemage. A threshold value is used to identiy those ponts in the region, which will be stored in a wo dimensionalaray rep resenting the global diplacement fid, macoming tor the rotation of larga regions, we apply sequence of small thite rotatons to all pontw othe test image. We can ill the boundary elements of the test inage the orinal the mage contans pixels onside the $512 \times 312$ region; oth ewse we hill in by zeros. The proces then ropeded bor the rotated res image, thereby bulng up the displack mentunctions. Ar the end of ampletertan sequence. gaps in the displacemen array are hlled in, as hn the case of sereo matching by a fnitedherencedfusion approx mation. Wh algorthm is much more computer intensive than stereo matchin. At the the of his withy only the global comelation and single thandion hav ben con pleded. The investgator are in the process of asseshng the size of resus that an be delected wh the global or relation funtions. Fis, 15 shows two neaty lentical vews of Banks lsand, localedromenst olaska, laken by basat with a 3 day inerval The loca homontal and verted correlatonvans artalculated after petoming globaloros corretation and translating the test mage by an amoun givon bye corration peak In Fg. 16 and and the hrge solid dak regins represent small values of homyntal and vertcal doplacements as one expects ater the test image ha been thastated. Fg. Tho shows the maximum loca corelaton value at od plxel hdratng a good math in the aneas wh a bwamoun of trangation. Theplxels in this regon conthited the loca mamum in the global or. retan tumetion

In adtion to sea-ice maphn, he sheme has recrety

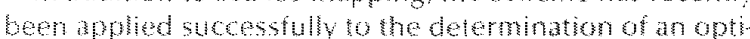
al tow hel tor the gan ted spot on lupiter, working as expected thus hat and thols extreme promse of being reasonaby sucessul tor the automatc moton dete. lon aplicalins

\section{Chemolly Penturbed Almospheric Modelngt}

If is well known that the actwites of humans are leading to importan changes in the composition of the earh's atmosphere. For instance, increasing amounts of carbon doxide ontribute to the greenhouse effect and are axpeded to lead to troposphetc waming and stratospheric ooling in the stratosphere increasng amounts of

The results presented in the sethon are based on work per wmen and material provided by Kave and R. Rond Labnatory

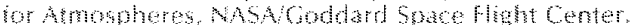




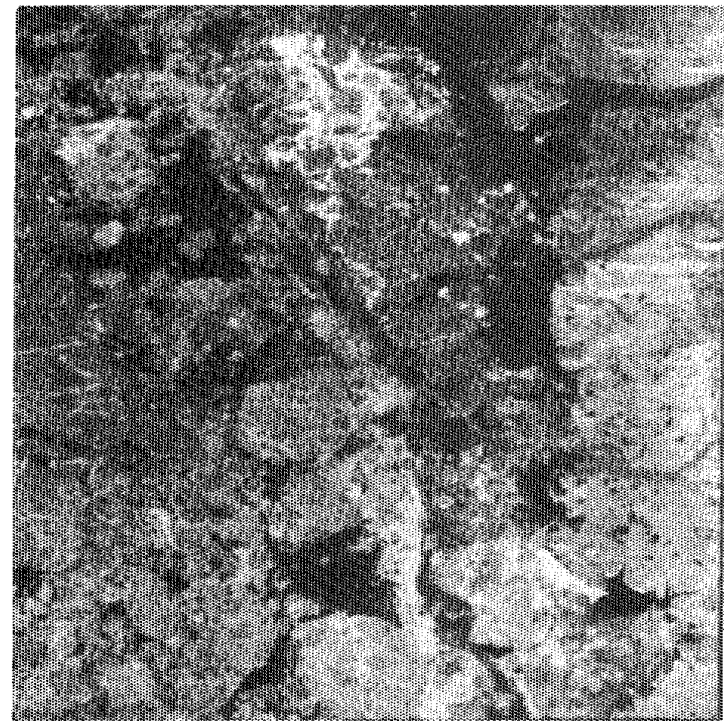

(a)

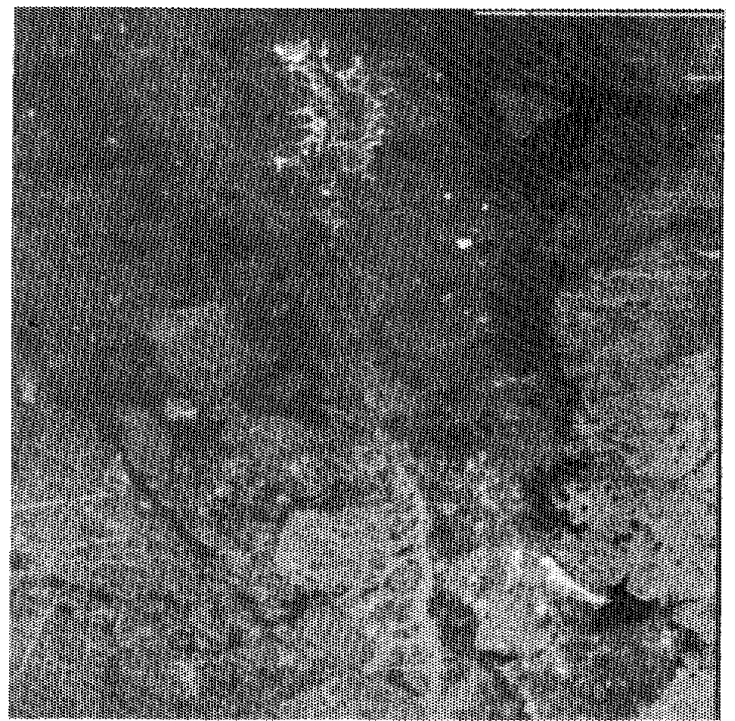

(b)

Fig. 15. Nar identica wews of Bank Isand, Aaska, van by Seast symhen aperure rakar ta was taker 3 days betore bl.

manmade horinecontaining substances are expected to lead tolarge decreases hozone. Chomecompounds have been definitely implicated in the Antarcic onone hole, and may alsobe responsible for part of the ozone decrose over the norhern hemisphere in the last decade.

Threepmensional statospheric Models: Comprehensive computer models that represent meteorology, chemistry, and radaton, and the interactions between them, are necessary to assess changes in atmosphertc composition. Such theedmensional models sat with the fuld dyamic equations for the atmosphere, but also explictly include revant chemical and photolytic reactions as part of the radlative processes. In addition, the model includes the conimuty equations for the chemical producton and loss of all relevant species. For a complete representaton of atmospherc chemisty, more han 25 ementsareneded. More than 60 reactions and photolysis rates are needed.

The equations are coupled nonlinearly becase the production and los rates depend on the values of the other constivents. This coupling is further complicated by the range in chemicallifimes of the constuents. Lietmes range from several years for nitrous oxde lo a lev days for hydrogen chloride to less han a second lor reactive intermediates such as elecironicaly excted oxygen atoms, spechal numerical lechnques must be used to imulhaneously solve these equations with widely varying the consants. The addion of chemistry to an atmospheric general cir culation model increases computer ime usage by an order of magnitude and memory requrements by a lactor of 5 . Given that the operaton of a midde atmosphere general circulation model requires the resources of a presentay supercomputer (CYBER 205), the additon of chemistry to the model is obvously imited by current technology.

Typicaly, either drastcaly reduced constiment sets must be used, or the spatial resolution of the model must be degraded seriously, Indeed, all the assescment calculations run to date to predict the response of the atmosphere to changing amounts of chorne and ofher trace gases have been rum wh wodmensinal models that do not even atempt to account for longitudinal efects.

The curent heedmensional chemistrytransport model under development at NASAIGSFC has both ser. ousy reduced spatial resolution (approximately go batude by $10^{\circ}$ longitude by $3.8 \mathrm{~km}$ verical and a lmited chemical constuent set 12 conslifuens. Even wh these restric tons, the threedimen sional modes have been used in simwations on the lime scale of wees only.

spatally, one would like a model to have a resolution on the order of $2 \circ$ in both lativude and longtude and $2 \mathrm{~km}$ in the vertical drection trom the ground $1070 \mathrm{~km}$. This cor. responts to and of $180 \times 90 \times 35$, or in txeces of 500000 grd points for each constituent. There are theoretical and numerical experments suggesting that twice this resolu. tion may benecssary. Chemical reaction rates must be calculatel or appromatud tor each gid poin and time step. sophisticated numencal techiques are also required lo ensure that the algorthm used ro solve the continuity equations does not produce negative constituent amounts. Time seps an be a tw hours at the most, but $15-30 \mathrm{~min}$ time steps are more approprate if dumal effects are consid. ered.

Mamy ot the fud dyamical aspects of the model can be vecorked lo lake advantage of cument computer archrectures. Howeve, chentcal and radiative processes are not as conducive to vectorization. The photolysis rates are particulary ditfoulto calculate, as they require knowledge of the local ultavolet radiation field, which in tum depends on he atmosphentc compostion above any grid point. This is a dificult process to vectorize, but it is deally suited to paralde processng. In summary, efficat, accurate mod. eling of global atmosphenc chemistry is dependent on increased compurer capabilites and the development of fast porallel prowesing machnes.

The Wreedimensional hem wry-transpor mode under 


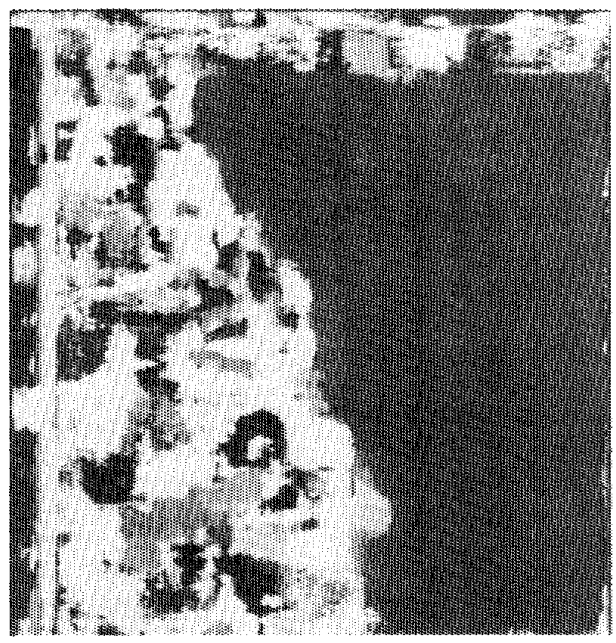

(a)

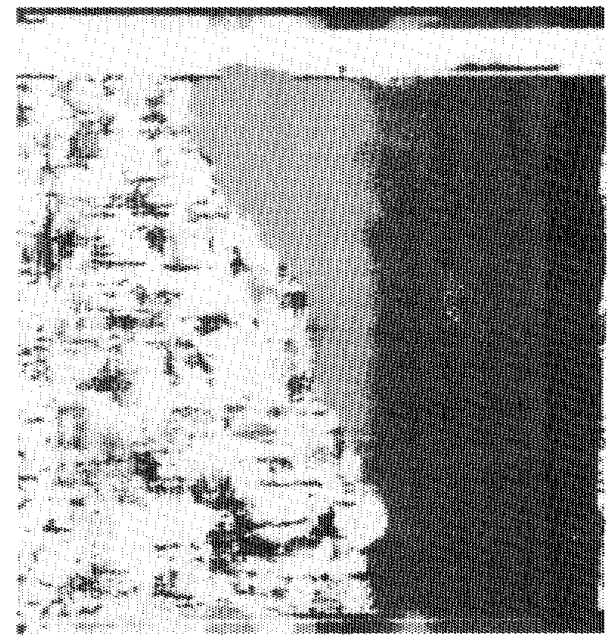

(b)



(c)

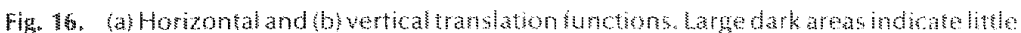


matches within overlapping ice loes.

developmen at the Laboratory for Atmospheres, NASA GSF, has the potential to lead to dramatic increases in our understanding of these issues [32] 134 ]. Using wind fields generated as part of an intenational global wather exper. iment held in 1979, the simultaneous efects of chemistry and transport wil be sudied for most important stalo. spheric trace constituents, including thoce containing oxygen, hydrogen, nitrogen, and chlorine. Carly eforis in this drection have locused on the time period in which the limb Intrared Monitor System (LMS) instrument on Nimbus 7 was operating so hat model calculations can be compared with satellie data.

A simulation sidy for the month of February 1979 was conducted with this model. Resuls indicate that the strucve of the computed constituent fields qualiatrely agrees wh LIMS observations. The temporal variance, caused largely by transport, is represented accurately. The good agrement between modelcomputations and globalobser. vations leads to the condusion that the model properlysm.
Whates the variablity of unobserved for less frequenty observed species.

bome of the longitudinal varablity calculated may be seen in Fig. 17 , in which contours showing the $\mathrm{O}_{3}$ distrbution at 18 mbar (topland the HNO, distribution al 18 mbar bottom) are plotted in a color scheme in which yellow is hgh, blue is low, and red is in between. Both $O$, and HNO; are longlived constituents at these pressure levels, especally at mide and high northem latides, so that dynamic processes should be reflected in their distributions. The high corratation beween 0 and 1 NO in the nothern hemisphere demonstrates that both are good racers of stratospheric motion at these locations. The transport of high.O, and low.HNO air nothward at the west of the regon near $180^{\circ} \mathrm{E}$ and $60^{\circ} \mathrm{N}$ and back southward east of if is cleary visible. There is itte longitudinal varation in the southem hemisphere, in which it is summer and the strat ospheric waves are small.

Results for species for which there are only very limited 


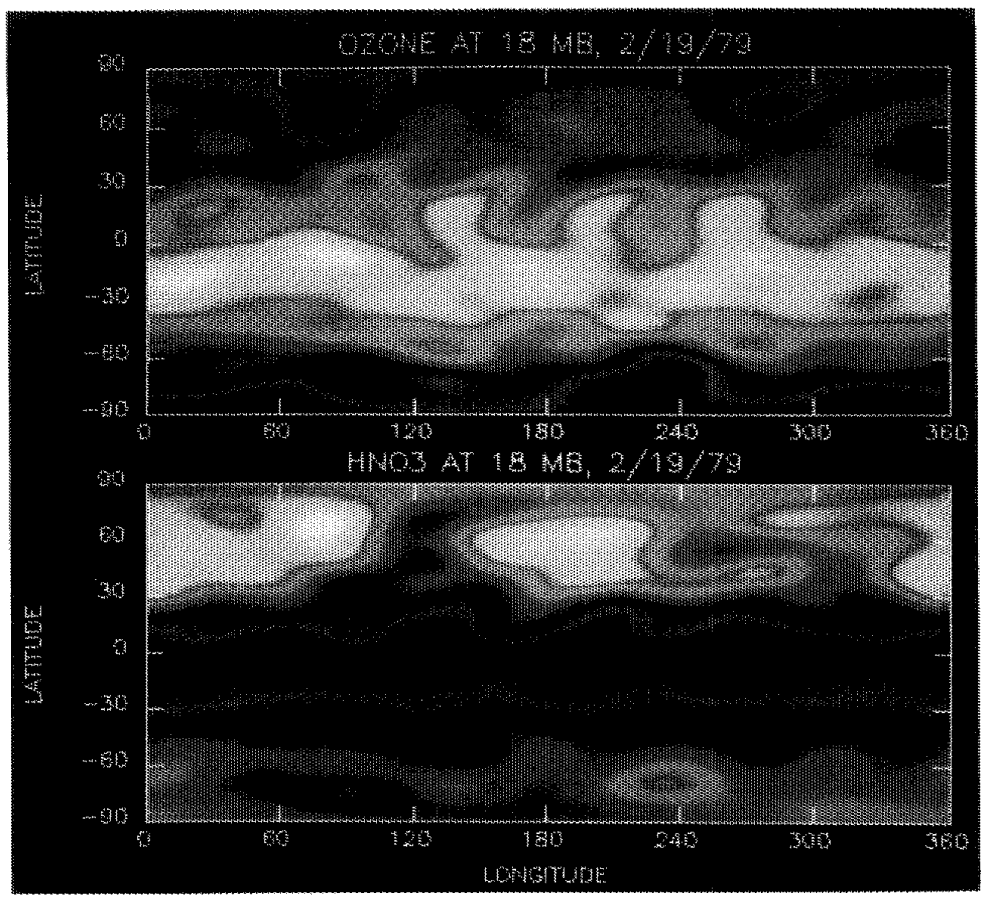

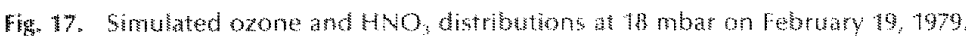

satellite based observations are shown in 18 , 18 , wh chlorine nitate $\left(\mathrm{ClONO}_{2}\right)$ and dinitrogen pentoxide $\left(\mathrm{N}_{2} \mathrm{O}_{5}\right)$ being in the top and bottom panels, respectively. The distributions are shown tor the 18 mbar pressure level where, except at high lantudes (polar night and polar day) both species underso important diumal varation. They maxi. mize at night and minimize during the day due to solar ullaviolet photolysis.) The distributions are thus expected to rellect boh diumal photochemical and dynamic varia. thons, and both may indeed be seen. In the cloNO, field. the diumal variation is seen most clearly in the high (red) values near $180^{\circ} \mathrm{E}$ and $60^{\circ}$, while the dynamic variation is

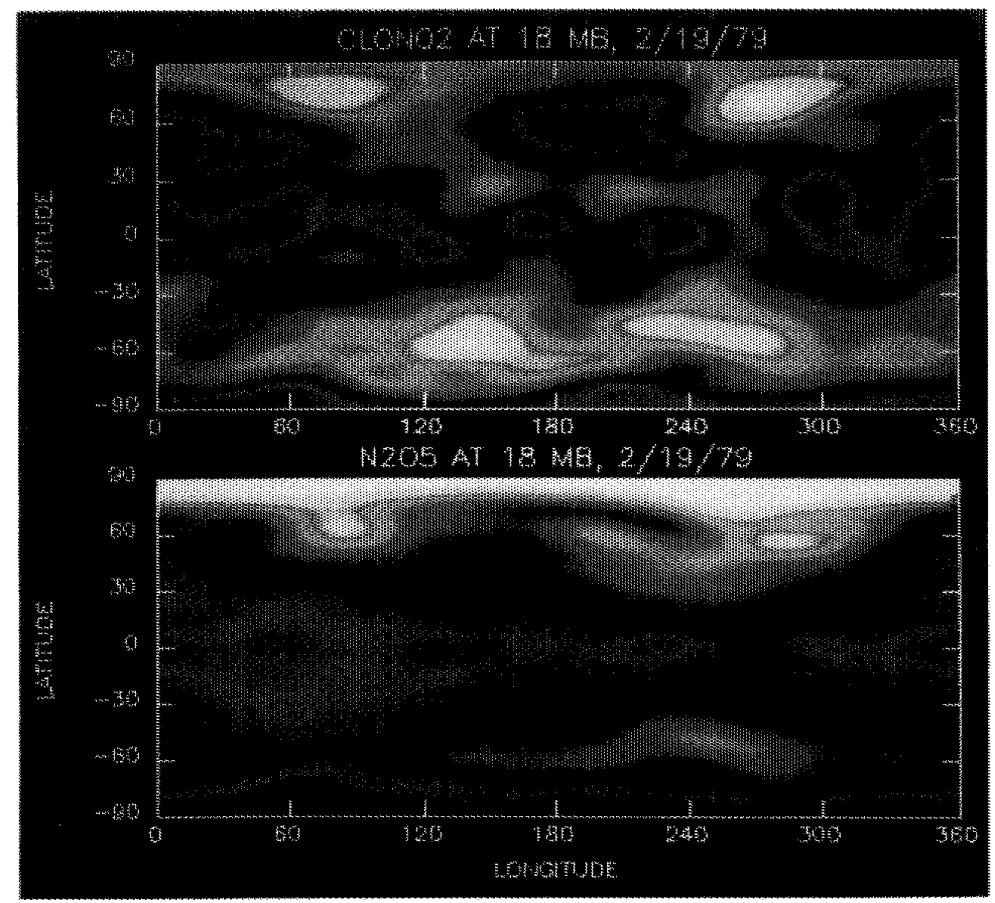

Fig. 18. Simulated $\mathrm{ClONO}_{2}$ and $\mathrm{N}_{2} \mathrm{O}_{3}$ distributions at 10 mar on February 19,1979 


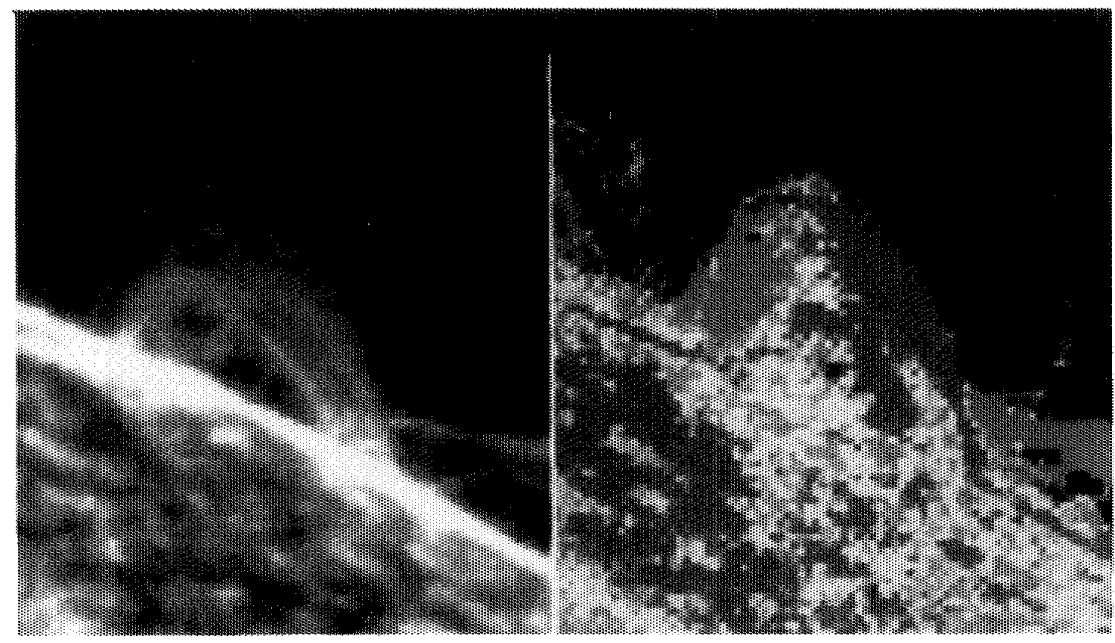

Fig. 19. Solar maximum mission ISMM observations of the intensty of a war flare loop and calulaton of the velocity of he same loop to provide boundary conditions for sim uating the physical processes occuring on the sth.

seen by the crescent-shaped region of high (yellow) ClONO air around the region of lower values. For $\mathrm{N}_{2} \mathrm{O}_{3}$ high values associated with the diurnal photochemical variation are seen centered near $240^{\circ}$ E over all but the most nothern lat itudes, while dynamic variations are seen at high northern latitudes by the two regions of enhanced values centered near $60^{\circ} \mathrm{E}$ and $270^{\circ} \mathrm{E}$.

\section{E. Solar Modeling of Flares and Coronal Heating}

Solar flares are enormous violent explosions of superhot gases covering billions of square miles on the surface of the sun, raising temperatures millons of degrees in seconds, dramaticaly increasing $X$ ray emssions and spewing bit. lions of tons of solar matter deep into space. Lasting only minutes, the effects of flares can be seen and lelt for hours, and their impact on the earth and its emiromment can be observed for days. Current knowledge of solar llares is lim. ited because flare processes cannot be duplicated in the laboratory. Launched in 1973, the Solar Maximum Mission (SMM) satelite carried six instruments ino space, enabling scientists for the first time to monitor solat flares (Fig. 19) occurring simultaneously in diferent wavelength regimes.

What triggers these explosive outbursts? What processes deep in the sun are responsible for releasing such tre mendous energies? Can solar flare activity be predicted using observational data? To date, linle success has been achieved through modeling. Recently, however, with the advent of supercomputers, models have exhibited some degree of success in simulating the solar flare phenome. non.

Hybrid Codes for Flare Modeling: It is well established that currents flow along closed magnetic field lines, which link one pointin the photosphere to another by first flowing up into the corona and back down again, that is, a closed loop. Alternately, these "field-aligned currents" may flow

The results presented in this section are based on wotk per formed and materal provided by $D$. Splce and $P$. Max Neice, Lab oratory for Astronomy and Solar Physics, NASAGoddand Space Fighn Center. along open magnetic field lines from a point in the photosphere out to "intinity." It is these field-aligned currents that are widely believed to be the source of free energy for solar flares and possibly the cause of coronal heating. Theory shows that there are three mechanisms that can extract the free energy stored in field-aligned currents. They are double layers, field-aligned anomalous resistivity, and / driven reconnection [35]-[42].

Ficldaligned electrofynamic coupling is a relatively new concept in solar physics [38]. An example that illustrates the defintion is that of an erupting filament. When a flamert erupts, it can interact with neighboring magnetic structures and, in so doing, perturbs the magnetic configuration. This perturbation can cause a variety of things to happen within the contiguration. Alven waves, in the form of tield-aligned currents that are cospatial and antiparallel, could be launched and nonthermal particles could be accelerated. Both of these effects are electrodynamic in nature because one involves field-aligned currents and the other involves the transport of charge particles. Both effects will propagate away from and toward the lower atmosphere, and during their transit, they both can modify the local plasma by changing the transport coefticients, which in turn can lead to local electric fields capable of accelerating particles. Those Alven waves and nonthermal particles that reach the lower atmosphere and refiect (or mirror) there will move back toward the original disturbance, but while making the rip can again modify the local conditions in a highly coupled and complex manner. To treat such a complex sequence of events analytically is impossible and is even diticult numerically. However, it must be done if theory is ever to properly support experimental research.

This coupling can only be modeled successfuly if proper account is aken of the kinetic behavior of the differen patticle species in the plasma. This requires the use of particle codes, that is, codes that follow the individual tratectories of a representative sample of particles in the plasma.

A hybrid model was developed which does just that. The ion dynamics in the hybrid code are treated using a particle. incell technique [43], [44]. The ion distribution is advaned 
in the under the infuence of a self-consisent Lorentzforce plus gravity using a simple leap frog technique for advancing the particles' postion, magnetic fielo aligned momentum, and pich angle. The relevant singlepartide equations are obtarsed by making the approximation that the ion Lar mor orbit is gyrotropic about the magnetic field line and the ambient magnetic held doss not change appraciably across an ion gyro radius or durng an hon gyro period. The resulting ratativtic particle equations are called he guid ing center equations [45]. The effect of collisional scatering on the ions is acheved rhough the apolication of a Monte Carlo scattering algorithm. This is based on the drag lor dynamic frichon ard velocify dimusion conffichents [46]. [47] for a charged test particle taveling through an ionized Max. wellian plasma.

Because the flectron plasma trequency is so high, the electron component of the plasma cannot yet be modeled using the same particle in-cell techrique withou the wse of prohibively small infegration time steps. Instad, it is described as a fluid that is coupled to the ion particles.

As a test case, an ldealized loop was set up with untorm electron temperature at one million kelvins in hydrostatic equilbrim, A total of 100000 particles were used to rep resent the ion component. These were loaded with a valoc iy distributon with the same temperature as the electrons, axcept near the loop lop, where $10 \%$ of the ion mass was loded whin a temperature of 100 millon kelvins. The hybrid modelgyrowaverage kinetic equations plus Monte Carlo scattering for ion particles and vavier-sokes sumation for electrons is integrated for abou 35 seconds. Fg. 20 shows the velocity distribution of a random sample of 2000 par ticles from the hoter ion component near the top as a func tion of their position for $=0,1.8$, and 2.7 seconds. Resuls show the expansion of the hotest componentrom the loop top whin the faster particles at theleading edge of the expansion. This calculation took about min on a CAY-2-about 100 times slower than real time for this number of particles.

These hybrid codes should have a signiman impact on the theories of solar flates, coronal heating, solar wind, and the electrodymame conpling of the photosphere to the corona since they allow computation of the spatial and cemporal evolution of either the ions or the electrons and bons within a lux tube, be closed like a loop or open like a coronal streamer.

\section{F. Formation of Stars}

Star fomation begins from a dense clump of gas embedded in molscular cloud. When the gravtational potental of this clmp or core becomes sufficienty large, the surrounding molecular gas begins to tall loward the center. slowly increasing the mass of the core. This accretion process continues unt the protostar and the resulting accre. tion system begins to drive an outflow of mass that eventually stops the accretion process and disperses the surrounding gas and accretion disk materat. Thus the observed pentod of mass outfow mepresents the transihonal phase of evolution between a purely accreting (growing protostar and a "naked" pre-main-sequence (newbom star (Tig. 21).

This phase of mass outhow plays an imporant role in the evoution of a protostar, providing a means of lurning of the mass accretion and, hence, determining the final mass of the star, in addion, these outflows have a significan effect on the paren molecular cloud, possibly influencing future star fomation within the clond.

About 3 to 4 years ago optically observed lets in contrast ro jets detected from radio wave emissions, were discovered in regions of active star formaton ofen associated with Herbig-Haro objects (hat is, dark condensation in bright emission line regions). These optical jess emanating from young, lowmass stars, along with molecular outflows, Her big-Haro objects, and strong stellat winds, are manifes tations of this phase of very energetic mass election that occus during the fomation of most, if not all, stars. Detaled models and observations are proving us with clues to the formation and evolution of these highy callmated jes and their relationship with other signs of mass outlow. As an example, recent numerical work is presented below, show ing that the enigmatic Herbig-Haro objects are simply the optical mantestations of the working surbace of radiatively cooling jets, in much the same way that optical flaments in supernova remmants are signatures of the blas wave hom an carlier supernova.

Stelar lat Modeling. The occurrence of jet streams, nar. row elongated features, embedded in dynamic or mag. netohydrodynamic systems, is one of he more remakable processes discovered in recent years. In ammospheric science, jet strems are thought to be responsible for gov eming the midatude weather circulations. In the oceans. jet currents like the Gulf stream are responsible for trans porting heat from equatoral regions poleward that are though to account for the moderate high-lathude European climates. In astrophysics, extragalactic radio jets, observed usmg the very-large-array radio telescopes, and their occurrence have been associated with active galactic nuclei. More recently, optical jets from molecular clouds have been observed with highresolution $\mathrm{CCD}$ images and as conjectured above, are though to be responstble for controlling and determining the final mass of the star. The universality of the dyamic mechanisms govenning these vastly difering astrophysical processes as basic manifes tations of the same phenomena has been described beavtifully by koengl 48 .

Optical jets were first discovered in regions of active star formation ony about 3 or 4 years ago and are often associated with Herbig Haro objects. Some hactsassociated with these stellar jets are 1 they are detected in high-resolution optical CCD images and by radio continum measure menis, 2) outlows are ypically collimated within $<10^{15} \mathrm{~cm}$ from the origin to an ppening angle of $3-10^{\circ}$, and 3 yeir velocities are on the order of $100 \mathrm{~m} 400 \mathrm{~km} / \mathrm{s}$.

Among the most recent exampas of Herbig-Haro $\mathrm{HH}$ oblectsare $H \mathrm{H}$ and $\mathrm{H} 2 \mathrm{2}$ in the Orion molecular cloud and the HH34 object [49]. Fig. 22 is a montage of wo CCD inages covering the HHA region. Here two bow shocks can be seen, one north of the source, labeled $H+34 \mathrm{~N}$, and one south, HH345. Several models have tred to acoount for some of the prominen working surface characteristics that is distont knots of emission win diferent radial velocities and faled. In this saction, we report on a successul detaled numerical simulation of radiative jets 150$]$ that links the "knoty" emission of Herbig-Haro objecs with the heads of stellar jets.

mhis section presents results obtained by Blondin aboratory for High Eergy strophysics, NASACoddard Soace Fight Center. 

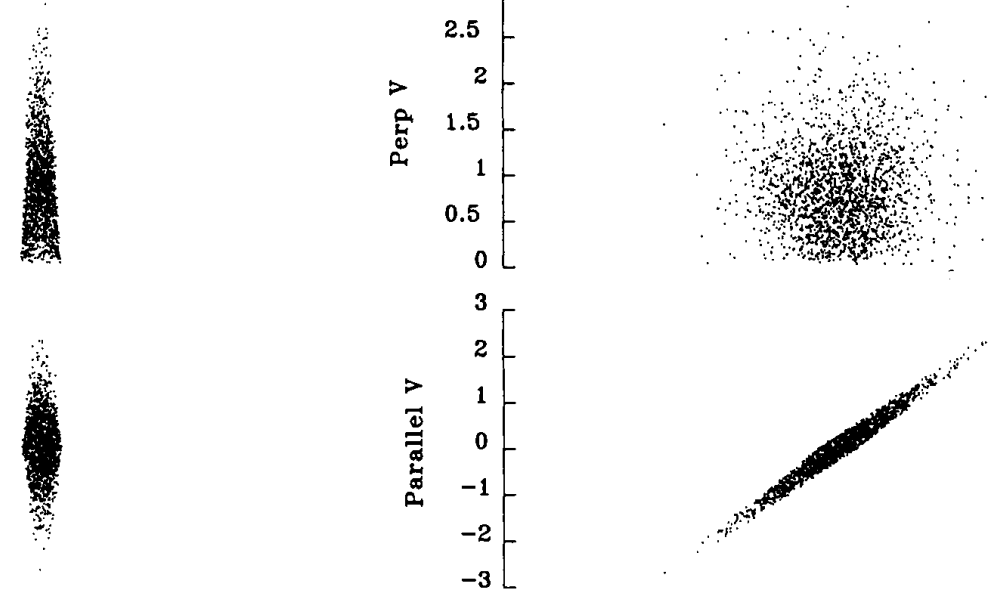

Height(cm)

(a)

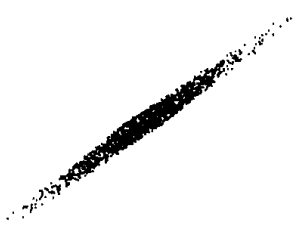

Height(cm)

(b)

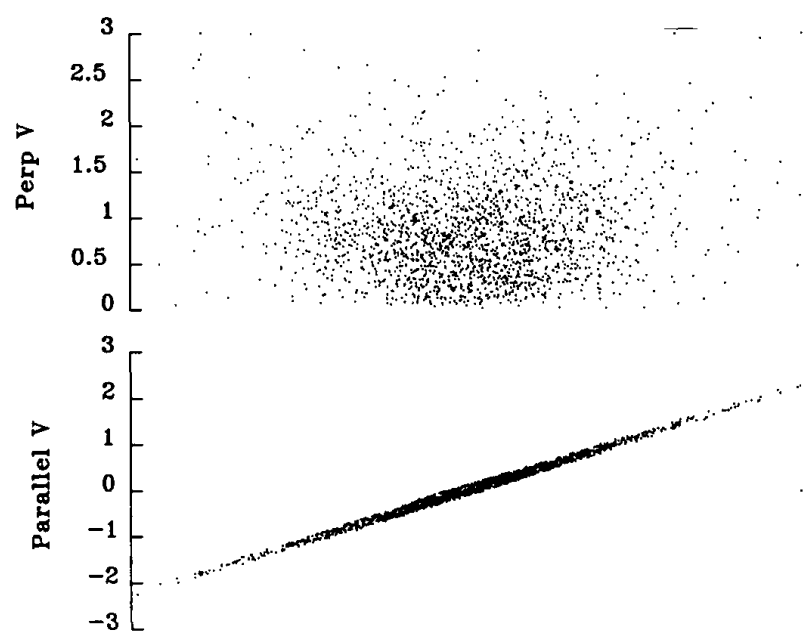

Height(cm)

(c)

Fig. 20. Plots of paraflel (magnetic field aligned) and perpendicular components of ion velocities in a flaring coronal loop simulation, calculated using a hybrid code (particle ions per fluid electrons). Hybrid code, phase space. These ions are streaming out of a rapidly heated section at the top of the loop. The hybrid code allows us to model kinetic effects such as magnetic mirroring of the ions in a converging flow tube. Typical simulations can follow the trajectories of a million or more ions. (a) $t=0$. (b) $t=1.8$ seconds. (c) $t=2.7$ seconds.

A two-dimensional gas-dynamics code developed by Blondin et al. [50] and based on the piecewise parabolic method [51] was used to study in detail the structure and evolution of radiatively cooling jets. The energy loss rate was treated as a local function of density and temperature only. This restriction is necessary to keep the computational requirements within the available computer resources. By not following the ionization state of the gas or the transfer of ionizing radiation, the cooling function in some parts of the postshock region may be underestimated by as much as an order of magnitude [52]. To approx- imate the nonequilibrium ionization, a cooling function is calculated for a gas cooling from $10^{6} \mathrm{~K}$ [53]. Using a cylindrically symmetric grid with a perfectly collimated beam injected along the axis of symmetry, the simulation employed a resolution of 30 zones per jet radius. The example presented here corresponds to a jet that is injected with a Mach number of 20 , a velocity of $250 \mathrm{~km} / \mathrm{s}$, a density $n_{\text {, }}$ $=20 \mathrm{~cm}^{-3}$, a density ratio $\eta=1$, and a radius $r_{j}=2 \times 10^{16}$ $\mathrm{cm}$. For these parameters we find that $t_{\mathrm{cool}} \approx 110 \mathrm{yr}$ and $t_{\mathrm{dyn}}$ $\equiv r_{i} / v_{i}=25 \mathrm{yr}$. These values may be representative of the jet head in $\mathrm{HH} 34 \mathrm{~S}$ [49], but the results should also be valid 


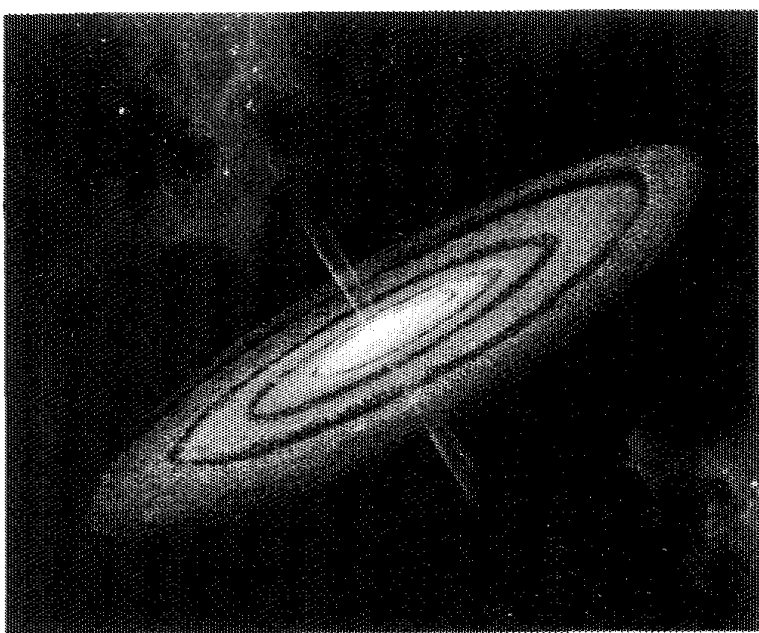

Fig. 21. Wis arist oncepton of a newhom stat sill hat

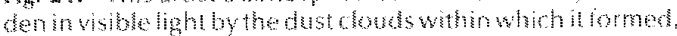
show mater in orbit around the rolating star such lefover debrimay eventrally form comets, planets, satelintes, and asteroids.



Fin. 22. Nontage of two CO imases of the HHzat region. for other soure parmeles provided hat they are scaled in such a way as to keep $X$ approximately consant.

Fig. 23 shows hedensty dotributionand shok strucure at the "woking sulace" of this jet alter l has propagated for approximately colling times. A dense shell of cold gas has formed and beromes progresively more massivas the iet contines lo advance. The evoluton of the "wotkng surace" regon show the rapid defomaton and fragmentation of the shell. Athough the evolution of a cooling Ia is detemmed by a complex interacton of votes shed ding, internal bean shocks, thermal insablites, and the dyamic instablity of the shall, we highighed a few key factors in this oxample that contribute to bet bavor.

Incomparing Fig. 23 with the observed mages of HerbigHaro oblects, if imporlant to bear in mind that a real on flows in general, neither homogeneous nor pertecty axsymmeric, and that the tendency form fistinct clumps would be whanced in aly thredimencional fow fig. 23 thus strongly suggests the possbinty that the knomy and

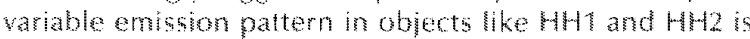
aconsequence of the dymmonstablin in we dense shels at the heads of the corresponding jets.

The simulations indicate that the standof distance of the "working suthes" shocks from the intervening dense shell change with time. This may be at least party related to the fact that radiative shocks with velocilles $y_{s}>150$ $\mathrm{km} / \mathrm{s}$ are subject to overstable oscllatons [52]. Eventhough the shok velocies in ou example $(<125 \mathrm{~km} / \mathrm{s}$ ) aro below this theshold, the shoks are still unlkely to be lound in a steady biale

The general propertes of Herbig-Haro objecls likeHH34 that appear to be associated with the hads of stellar lets can be reasonably well reproduced by our nonadabatic ie model. This model predis that dense shell should fom between the jet shock and the bow shok atter the columm densiv of the radiativly coong gas becmes suficienty large, regerdes of which of these wo shocks is more strongy radiative that 1 , regardess of whether the density ratio is iscater or smaller than 1 . The shel is highy un sable irrespective of the ambient densivy distribution. exhbing large structural varation on the sales as shor as thm and is expected to eventualy fragment into separte clumps. These clumps, which ocastonaly acqure a sig. nilont transerse veloty component, ma be identifed with the distinct emission knots observed in Herbig - Haro


surace" may aso be associated whoverstable oscillations of the adiane hocks as well as whth the lormation of iso lated condensalions by local thernal instabilty. In add ton, any denseclouds that have been entained the low and carred the let's head would undergo ballstic de ceterationater crosing the jet shok and may be purcened "s separate "interstelar bullets."

A comparson of models with observatons of stellar jets will halp constrain estmates of the mass and momentum fux in the fer wel as the density and pressure he the sur. rounding gas. This inomation can then be used hetrer understand the mass and energy budget of the acereting protontar that is dring the jet. Future studes, both the orebal and observational, will focus on the question of let tomation, which occurs very close to the acerting star. These stellar jets have been detected with high-resoluton optical CCO images and by ratio continum measue. 


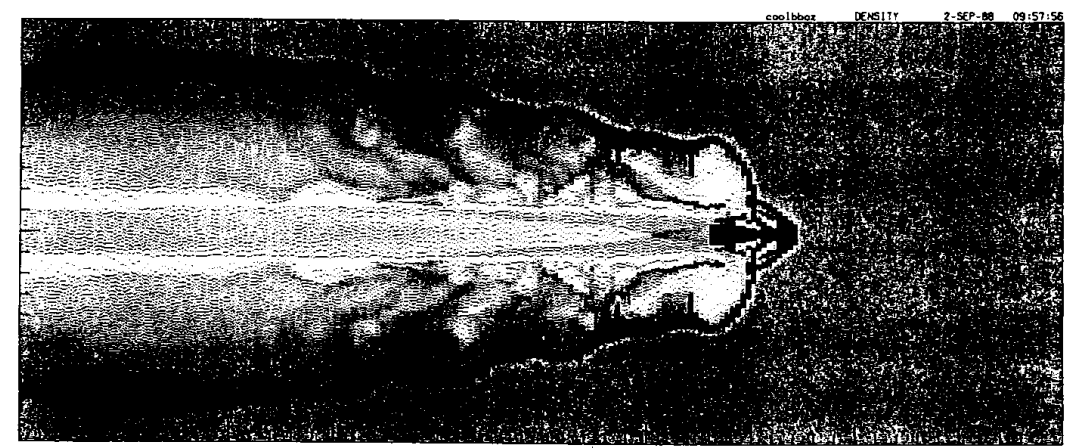

Fig. 23. Density map of the "working surface" region for the simulated jet after 1400 years of propagation. The jet head is advancing at approximately $125 \mathrm{~km} / \mathrm{s}$ and has reached a distance of $5.7 \times 10^{17} \mathrm{~cm}$. The shading in this plot varies continuously from white (zero density) to black (saturation level, corresponding to a density of $350 \mathrm{~cm}^{-3}$ ), with the ambient density being $20 \mathrm{~cm}^{-3}$.

ments. Outflows are typically collimated within $10^{15} \mathrm{~cm}$ from the origin to an angle of $3-10^{\circ}$ and have velocities on the order of $100-400 \mathrm{~km} / \mathrm{s}$ scales similar to the size of the accretion disk. By understanding the formation and collimation of these jets, we may learn more about the geometry of the accreting protostar and disk and about the trigger that begins the mass outflow phase and ends the mass accretion phase. A complete understanding of jet formation and evolution is thus intimately tied to the dynamics and evolution of star formation.

\section{G. Imaging the Cosmic Background Radiation}

Sometime around July 1989 NASA will launch the Cosmic Background Explorer Satellite (COBE) in an attempt to detect the radiation emitted from the first objects or structures to form after the Big Bang. In addition, COBE will determine whether possible distortions or small anisotropies in the 2.7 $K$ cosmic microwave background exist and will permit the study of other sources of diffuse background radiation at wavelengths from $1 \mu \mathrm{m}$ to $1 \mathrm{~cm}$. Other sources could include interplanetary and interstellar dust, hot electrons in the galaxy, faint stars in the galaxy, and possibly infrared galaxies and hot gas in galaxy clusters. In order to map these primeval and local sources, COBE will scan the sky repeatedly with its three instruments, building up signal-to-noise ratios until the data are only limited by the astrophysical environment. COBE's three instruments are the diffuse infrared background experiment (DIRBE) covering $1-300 \mu \mathrm{m}$ with a 10-band filter photometer and a $0.7^{\circ}$ beamwidth; the far infrared absolute spectrophotometer (FIRAS) covering 100 $\mu \mathrm{m}$ to $1 \mathrm{~cm}$ with an absolutely calibrated polarizing Michelson interferometer with a $5 \%$ spectral resolution and $7^{\circ}$ beamwidth; and the differential microwave radiometer (DMR) covering $31.4,53$, and $90 \mathrm{GHz}$ with $7^{\circ}$ angular resolution. Alf three instruments depend on spectacular advances in the sensitivity and instrument accuracy and are expected to lead to the discovery of new phenomena in the early universe. The technology to analyze these data relies on intensive interactive computer analysis systems eventually requiring specialized supercomputing systems. A short background description of the astrophysical data analysis system developed for these studies is described below.
Astrophysical Interactive Computer Analysis ${ }^{7}$ : Since the invention of the telescope, the classic problem in optical astronomy has been removing the effects of the terrestrial atmosphere from astronomical data. During this century, optical astronomers have learned to carefully correct for atmospheric absorption and estimate the effects of clouds. In the process, they have discovered interstellar reddening, interstellar clouds of dust and gas, wavelength variations of extinction (reddening), variations in interstellar dust parameters as a function of position in the sky, interstellar polarization, magnetic fields, and so forth. Even when observing from space platforms, many of these effects must still be carefully accounted for, depending on the observations being conducted. Astronomy today uses twodimensional format detectors across the entire electromagnetic spectrum from gamma rays to radio wavelengths. To extract the full significance of any two-dimensional map of the sky in a particular wavelength, extensive interpretive studies with computer-intensive image processing techniques are required. Today astrophysical science is working at the very limits of measurements-attempting to extract very weak signals from noise in the presence of much stronger signals emanating from sources not under investigation. For example, depending on the weak source to be studied, confusing sources could be the earth's atmosphere, the interplanetary medium, the interstellar medium, the galactic signature, or even the intergalactic medium. Hence astronomical observations from space will require unwanted astronomical signatures to be removed from the data. Moreover, the instrument signature itself has to be perfectly accounted for in the data processing. Thus models of the sources of confusion are constructed and literally subtracted from the data so that the noise can be investigated for signals that might herald a new discovery about the solar system, galaxy, or universe. Interactive astrophysical modeling permits such investigations to progress at an unprecedented rate. This increase is necessary to comprehend efficiently and quickly the huge volume of data produced by modern space instruments.

\footnotetext{
${ }^{7}$ This section presents material prepared by J. M. Hollis, Space Data and Computing Division, NASA/Goddard Space Flight Cen-
} ter. 
Astronomers are becoming data bound as they acquire more data than they can analyze or process accurately enough to account for astronomical phenomena.

COBE has two "amospheres" with which to conlendthe interplanetary atmosphere and the galactic atmosphere. Both possess a basic symmetry with significant deviations. The brightness of interplanetary dust is a strong function of solar elongation angle and eclipticlatidude, and at short wavelengths it scatters polarized sunlight. The dust is approximately symmetric about the eclipic plane and independent of longitude, but the nomina symmety plane is distorted as it passes near the various planets. Streams bands in the dust are due to sources in asteroid family col. Ifsons, resonances with maipor planets spoil the rotational symmetry, and there are traces of the trals of comels. The interplanetary dust composition and size distribution may difer with the distance from the sun as well. Smilarly, gal. actic intrated emissions clearly have the general distribution seen at other wavelengths, with a concentration in a plane, a greater concentration loward the center, and an iregular (cloud distribution with variations on all angular scales from fine filaments to huge clouds and loops. The galactic dust temperature is, on the oherhand, a very slowly varying function across the sky.

All of these phenomena can be modeled numerically. There are many observations a many wavelengths to be explained smultaneously by a single model, so some complexity is required. On the other hand, there are fewer adjustable parameters than observations. Simulations have shown that it should be possible to model the interpla. netary dust emissions and subtract them from the observations with an accuracy on the order of $\%$. Similar accuracy for the interstellar sources is less likely because of the irregular cloud distributions.

There are also important concerns about the effects of possible erors in the instruments. The instruments have been designed to adequately avoid the known errors of other instuments of their $1 y p$. Nevertheless, some additional phenomena may have to be modeled with a computer. As an example, the terrestrial and spacecraf magnetic fields affect the DMR slghtly, charged particles add nolse to the FIRAS and DIRBE detectors, and the expected high solar actwity may rase the earth's atmospheric density to alevel where its emissions could be seen with the DIRBE. There is anallefect of the instrumen sids loberesponses to the mon, earth, and sun, particulary on the DNR data, which is amenable to numerical modelng.

The lmiting factor for the scientific value of the COBE observations will be our ablity to mode, explain, and subtract the local background sources and derive a residual of cosmic origin. Displaying these data and adjusting the parameters of models to fit them are key parts of the COBE mission. The COBE project has selected the astronomical image processing system (APS) as the basis for the final display and analysis. The procedures being developed wil permit integrated modeling, display, and analysis on a sin. gle computer with high speed and user convenience, enhancing the accuracyand value of the frnal products, and enabling the results to be oblaned more quickly. Fig. 24 simulates the kind of new displays and visualzation tech. niques being developed to visualize he data acquired from the Cobe project.

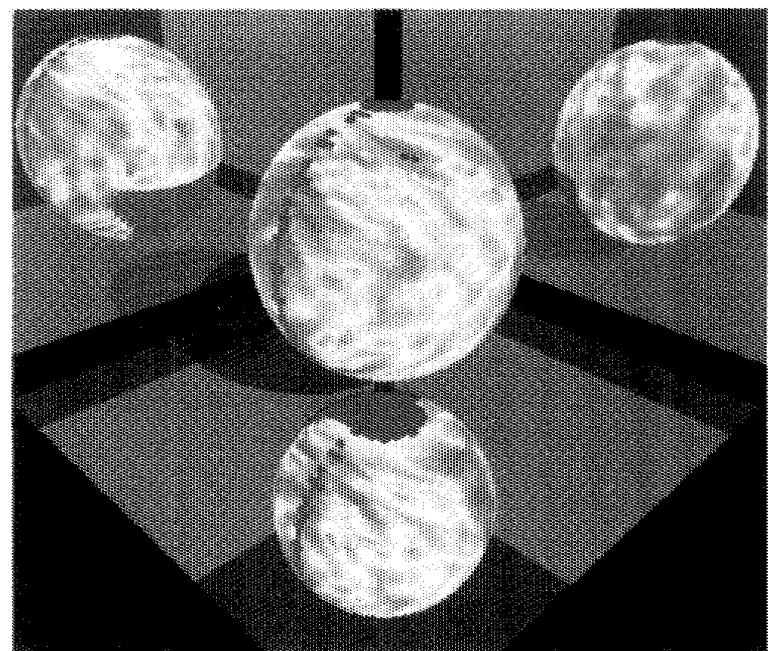

Fig. 24. New woy to visualize all.sky data. The sphere represents the cosmic backgound radiation anisotropy as the OMk will see it. Ths particular display principally shows the dipole aniotropy (red and blue shify due to the motion of the eath observer with respect to the cosmic bakgrothd rest frome. The thee mirros permit the viewer to see the whole sphere at once, without the distortion inherent in planar mapping, The graphiss MSWS will allow the scients to rurn the sphere will, to the dat in rablsme, and to display successive models or residuals.

\section{Mulmolscimenary Somatic Compline}

The expeciation of continued exponential growth of computing power and space data into the naxt century offers a grand challenge for earth and space scientific computing. We are already winess to the extension of classical discipline modeling (suchas atmospheric circulation, ocen dynanics, and star formation into the areas of detaled multidisciplinary simulation, such as ocean-atmospherebiosphere ineractions, mdiation-chemistry dynamics of the wpper atmosphere, and wil-scale modeling of the solid and liquid pats of the planet through the mantle to the lith. osphere. Simlar modeling problems occur in simulating the formation of galaxies where not only selt-yravitational interactions of stars need to be considered, but also the affects of moleculat clouds of gas. The need to consider mulidiscipline modeling occurs in solar and space physical phenomena as well. Interdisciplinary science involves chal. lenges in coupling distind disciplnes-themselves still under development. Dealing with the relaxation of prescribed boundary conditions or developing new parame. terizations of physical processes that have been very crudely considered will require a program of expermentation as exiensive as that which yidded our present modeling capability. Wenow oullne some of the future directons planned for the disciplne modeling efforts described in the previous section.

Toward a Core-Manle-Lithosphere Model. Neary $70 \%$ of the earth's mass is the region between the crust and the cone, known as the mante. The tormation and motions of the lithospheric plates (including the crust and the upper. mos mantel depend on the evolution and dynamics of the underlyng mantle. These plates are the cold upper bound- 
ary layers of mantle convection cells. Though composed of rocky silicates and oxides, the solid mantle is partially molten and convects like a fluid over geologic time scales. Mantle convection is driven both by the heat of radioactive decay within it and, in part, by the heat flux across the core-mantle boundary. Thus the forward problem of modeling the "solid" earth over geologic time scales involves the "climatology" of interacting viscoelastic-plastic-fluid systems. However, the fast time scales are associated with the liquid outer core (rather than the atmosphere), and the slow time scales are associated with the mantle (rather than the ocean), the lithosphere (rather than the icy cryosphere), and the inner core (rather than solar evolution).

One goal of "solid" earth modeling is to simulate the planet from the core through the mantle to the crust and to test the results of such simulations against observations. Developing the capability to attain this goal requires improvements in forward modeling, in the inversion of geophysical data, and in the geophysical data base. Individual forward model components for studies of plate deformations, midocean ridge and subduction zones, mantle convection, and the geodynamo must be developed, improved upon, and synthesized. Data inversion techniques enabling the estimation of parameters appearing in the forward models and properties predicted by such models also need development, improvement, and additional synthesis. The successful application of such techniques hinges on the acquisition of more, high-quality observations.

Undertaking a numerical simulation of the coupled coremantle-lithosphere system seems timely in view of 1) the possibility of acquiring crucial observational data from space and ground, 2) the feasibility of inverting such data for present-day earth structure, and 3) the limited, albeit great, success forward modelers have had in simulating the behavior of these domains separately.

It is hoped that by the mid-1990s NASA will initiate a geopotential research mission to provide the uniformly excellent data needed to determine the earth's gravitational field with very high accuracy and spatial resolution. GRM magnetic measurements would also update the 1980 Magsat determination of the main geomagnetic field, thus providing tight constraints on acceptable models of geomagnetic secular variation. Sustained monitoring of secular variation will hopefully be achieved by the geomagnetic observing system (GOS) on EOS. Together with an international program for augmenting the global network of seismometers and continued monitoring of the earth's variable rotation, these diverse types of geophysical data can be jointly inverted for a unified model of the coupled core-mantle system [3], [6]. Correction, or simultaneous solution, for lithospheric effects is a prerequisite for such an inversion. High-accuracy determination of the earth's surface topography from a variety of satellite altimeter measurements (TOPEX, Geosat, and EOS) will be useful for correcting the gravity field and thus for deriving a satisfactory present-day representation of the core-mantle-lithosphere system. The processing, analysis, and either independent or joint inversion of these diverse geophysical data types pose supercomputational problems as challenging and rewarding as those posed by a forward core-mantle-lithosphere model.

Coupled Ocean-Atmosphere Dynamics: The most prominent phenomenon involving the coupled dynamics of ocean and atmosphere is the El Niño southern oscillation
(ENSO). There is growing evidence from modeling studies that ENSO is a natural, self-sustained vacillation of the coupled ocean-atmosphere system. This natural variability has been studied with linear and idealized models [54], [55], with simple nonlinear dynamic models [56], [57], and with coupled ocean and atmospheric GCMs. It is clear from these results that a detailed understanding of ocean-atmosphere interactions will be necessary to explain the phenomenon, and that much development of coupled models will have to take place before we can use them to predict ENSO.

A theory that can explain this internal variability and predict the multiyear time scale of the phenomenon has been developed based on the linear idealized coupled models described [55], [57]-[59].

In this theory, the time scale is related to the propagation times of equatorially trapped waves in the ocean. Simple models that include their role in the tropical coupled problem produce regular oscillations of the appropriate periods. However, when factors such as the seasonal cycle or the influences of random weather events are included, the regular oscillation is replaced by a more realistic variability, irregular in both period and amplitude, but whose time scale is still controlled by the "underlying" periodic solution. In addition to the obvious implications for the predictability of the phenomenon, this result also affects the analysis and interpretation of the observations. The immediate problem in ENSO theory is to test and examine the simple theory in the much more complex setting of realistic coupled GCMs.

Tropical coupling introduces a new time scale to the climate system that is not found in either ocean or atmosphere alone. No such fundamentally new modes have yet been identified in mid and high latitudes operating on interannual time scales. The mixed layer plays a central role in this area. While large-scale ocean-atmosphere interaction through these processes has not received much attention, they may also result in natural low-frequency variability, which is of interest to EOS.

Ocean-Ice Interactions: In the polar regions, a key problem is to develop an understanding of the interannual variability of sea ice, which is an integral component of the climate system in the polar regions [60]. Exchanges between ocean and atmosphere of heat, momentum, and trace gases are strongly influenced by the presence of sea ice, which serves as an effective insulator between the ocean and the atmosphere. In the vicinity of the ice edge, monthly mean heat fluxes between the atmosphere and open water can reach values of $500 \mathrm{~W} / \mathrm{m}^{2}$ in winter [23], primarily due to sensible heat flux. This can be reduced by one or even two orders of magnitude in the presence of a sea-ice cover [58]. Sea ice also lessens the absorption of solar radiation at the earth's surface because of its very high albedo $(50-90 \%)$ relative to that of open ocean (5-15\%). Large-scale changes in the position of the ice edge and the compactness of the ice field can consequently have important influences on largescale balances of heat, momentum, and radiation and on the spatial concentrations of various atmospheric trace gases.

Changes in the ice edge can also be important indicators of more subtle changes in the oceans and the atmosphere, because local changes will be reflected in the ice edge position. The ice edge is readily discerned in passive-microwave data. Observations from the Nimbus 5 and Nimbus 7 micro- 
wave sensors have proven the capability of routinely monitoring global sea-ice distributions from space and have revealed important elements of seasonal and interannual variability in the sea-ice cover over the past two decades [59]-[61]. The microwave sensor on a Department of Defense satellite launched in June 1987 and future sensors planned for EOS should extend the continuous coverage with passive-microwave data sets.

Processes that determine the sea-ice distribution and compactness include the direct heat exchanges with the atmosphere, the combined stresses of wind and currents, and the supply of heat by the ocean through both horizontal currents and vertical mixing. All of these processes are intimately connected and influence each other. While at certain times, and in certain cases, one element may appear to "drive" the others, counterexamples can usually be found. Understanding the coupled dynamics of these phenomena taken together is of very high priority.

Ocean Biology and Chemistry: One of the major aims of the globalocean flux study (GOFS) is to determine the mean and fluctuating components of world ocean primary productivity and their relationship to physical processes. While GOFS is a pre-EOS program, the importance of understanding this relationship will remain for the EOS project as well. Biological activity plays a key role in reducing the partial pressure of $\mathrm{CO}_{2}\left(p \mathrm{CO}_{2}\right)$ at the surface, thereby influencing the flux of carbon from the ocean to the atmosphere and the global atmospheric $\mathrm{CO}_{2}$ budget. On the time scales of interest for the EOS program, variations in physical processes-upwelling and advection within the ocean, radiation through the atmosphere-play key roles in regulating such biological activity.

On scales large enough to imply quasistatic balances, the biological activity can be viewed in term sof the carbon budget, with deposition of carbon from the mixed layer into the deeper ocean largely balanced by new nitrate-supported production [62]. Since the primary source of nutrient is in recycled organic material from the deeper ocean, variations in upwelling turbulent entrainment of nutrient-rich water into the mixed layer will result in alterations in the production and use of dissolved organic matter. In addition, understanding the maintenance of high-nutrient water just below the mixed layer and the important processes at work is an important problem that must be addressed. This involves coming to an understanding of both remineralization of the sinking flux of carbon and diffusion and transport of dissolved nutrients.

The aim of coupling ocean biology and chemistry will begin to develop better understandings of these processes and indicate how large-scale variations such as EI Niño can be expected to alter the surface biomass concentrations.

Land-Atmosphere Interactions: The extent to which local land surface moisture conditions affect local climate cannot be answered fully and quantitatively through observations alone. In fact, different interpretations of available observations have led to conflicting statements in the literature. For example, some researchers infer that precipitation over land surfaces is largely derived from locally evaporated water [63], while others claim that most local precipitation water is derived from remote sources and therefore is not strongly influenced by local moisture conditions [64], [65]. It is extremely important to understand the link between land surface moisture and climate, since human-induced changes in land surface conditions such as deforestation, irrigation, swamp drainage, and rerouting of rivers are occurring over significant spatial scales [66].

The ability of land surface evaporation rates to affect the global atmospheric circulation was discussed in Yeh et al. [67]. An increased evaporation can modify the thermal state of the atmosphere by both cooling the atmosphere near the surface through a decrease in surface sensible heat flux and long-wave radiation, and heating the middle troposphere through an enhancement of precipitation. GCM studies have, in fact, found that land surface moisture conditions can affect at least local climates [67]-[71].

An important land-atmosphere interaction to be studied in future coupled model systems involves the growth of vegetation. As atmospheric $\mathrm{CO}_{2}$ increases, vegetation could incorporate a significant portion of the excess into plant tissues. Also, as regional climates become inhospitable to indigenous vegetation, vegetation types could change, leading to a change in surface flux characteristics. Incorporating interactive vegetation into our models would not only allow them to respond more realistically to changes in global $\mathrm{CO}_{2}$, it would also indicate where vegetation changes are likely to occur first, thereby suggesting where we should look first in real-world data sets (as collected, for example, by EOS) for evidence of global warming.

On-Board Image Modeling: Space-borne imaging sensors for remotely observing the earth will continue to evolve in capabilities and to add more massive volumes of valuable scientific data. Already, the planned capability of instruments for acquiring data on the EOS platform of the space station greatly outstrips our ability even to transmit all these data to our ground processing systems. In addition it is expected that scientists in the manned space-laboratory modules will want to carry out research campaigns on events of opportunity, requiring on-board image processors of extensive performance capabilities. As a result, studies are underway at NASA to explore new technologies required for greater on-board processing capabilities to capture greater amounts of potentially useful data.

Complementary metal-oxide silicon (CMOS), which has demonstrated a unique potential for remote-sensing applications, was based on the custom chip capabilities of the late 1970 s and early 1980s. The MPP array used 88 boards, each containing $24 \mathrm{CMOS}$ chips. On each chip were embedded 8000 transistors, allowing the design of eight complete processors that use circuits of $4.5-\mu \mathrm{m}$ line width. Adjacent to each processor chip on the board were two memory chips of 4 kilobits each with appropriate pin connections to the processor chips, thus providing 1 kilobit of memory per processor. Parallel computers with arrays of 16000 to 64000 processors are now commercially available at prices only slightly greater than minicomputers of the VAX-11/780 class. Parallel processors with somewhat smaller arrays and deeper memories are also becoming available at costs comparable with SUN or micro-VAX workstations. Clearly, parallel processing is becoming a way to go in the pursuit of new image processing capabilities.

In this regard, NASA-supported research studies have already produced chips containing slightly more than 1 million transistors, allowing for 128 processors on a single chip, with each processor containing over 1 kilobit of on-chip memory. Memory chips are now available with 4 megabits allowing for an additional 16 to 64 kilobits of off-chip mem- 
ory for each processor. Thus, well before 1995, when the space station is expected to be operational, MPP-like workstations on a single board should be available with an order of magnitude more power than today's MPPs. It is similarly expected that a fully space-qualified MPP-like workstation will also be available for research aboard the manned laboratory modules of the space station. In all probability, one could also expect to find at the large ground processing facilities parallel processors with arrays of more than $1 \mathrm{mil}-$ lion processors and internal memories of more than 1 gigabyte.

Thus the immediate challenge facing the remote-sensing community is not in the hardware arena, but rather the development of greatly improved parallel image algorithms that will exploit these new computing architectures to maximize the extraction and use of the full scientific information content of image data.

Atmospheric-Stratospheric Models: As pointed out in Section II-D, application of the stratospheric general circulation and chemistry model to long-term assessment problems is impossible in today's computer environment. However, three-dimensional models offer the greatest potential of understanding interannual and long-term natural fluctuations and their impact on trend studies. Currently a suitable role of three-dimensional models in assessment applications is being developed. The advent of larger and faster computers will allow the full use of three-dimensional models in this important undertaking. In the current computing environment three-dimensional models are just becoming available for data interpretation. Some applications are outlined below.

Space-based instruments have provided valuable information about the distribution of several important trace constituents in the stratosphere. For example, on NASA's Nimbus 7 satellite, the Limb Infrared Monitor of the Stratosphere (LIMS) instrument provided daily maps of the concentrations of ozone $\left(\mathrm{O}_{3}\right)$, nitrogen dioxide $\left(\mathrm{NO}_{2}\right)$, water $\left(\mathrm{H}_{2} \mathrm{O}\right)$, and nitric acid $\left(\mathrm{HNO}_{3}\right)$ for a 7 -month period (November 1978 to May 1979). Several other satellite- and shuttleborne instruments have made measurements of trace constituent amounts in the stratosphere, but provided less than complete geographical coverage.

The space-based data are complemented by ground-, balloon-, and aircraft-based measurements. These measurements do not have the spatial and temporal coverage of the satellite data, but provide information on many more constituents. For instance, there are no global measurements of any chlorine-containing constituent in the stratosphere, and there will be none until the launch of NASA's Upper Atmosphere Research Satellite (UARS), currently scheduled for 1991. Since chlorine-containing species are believed to play a crucial role in anthropogenic ozone depletion, this is a serious deficiency.

Knowledge of the spatial and temporal variability of trace constituent amounts is important to help us understand exactly how representative the individual measurements are. Furthermore, it is necessary to know both the natural variability of constituent fields and the effect of atmospheric motions on atmospheric chemical composition in order to confidently extract long-term trends in trace constituent amounts.

The three-dimensional chemistry model with winds from the assimilation of observations provides the best quan- titative tool currently available to understand constituent variability. Three-dimensional model interpretation of LIMS data has been discussed, as has the theoretical investigation of the variability of unobserved constituents [32]-[34] We anticipate that the model will be invaluable for interpreting UARS data. Furthermore, the model is being used as part of the Airborne Arctic Stratosphere Expedition (AASE, January/February 1989) to determine expected variability and the potential global effects of localized heterogeneous chemistry that might be important in ozone depletion.

Solar Flares: Hybrid models should be recoded for parallel (concurrent) processors and extended to three-dimensions to allow more realistic efforts of loops and to allow for more particles in the simulations. Second-order effects and the use of multiple time and spatial scales, including kinetic processes, need to be added. These models will greatly improve solar flare safety factors for manned space flight and will permit the understanding of basic solar processes at a fundamental scientific level.

Stars and Galaxies: Understanding the formation and the evolution of stars and galaxies involves major observational and theoretical efforts. Progress in our understanding of the star formation process and in stellar evolution is essential if we are to understand the life cycle of galaxies. The numerical investigations of galactic formation and evolution involve the complex dynamics of the gas and stars in the galaxies. The complex interplay of many processes requires the study of the nonlinear time development of systems in complex three-dimensional geometries. Numerical experiments, based on large-scale computations, are the closest thing we have to the laboratory experiments of other parts of physics.

The dynamic range required in numerical experiments translates to grid sizes of 1024 on a side and to memory requirements of more than a gigaword. These requirements are also being driven by the observational capabilities expected in the near future.

New software technology and algorithms employing recently developed multiple time-scale techniques will be essential to follow physics on these different scales. The time evolutionary calculations will demand interactive data display techniques to achieve qualitative and quantitative understanding of the results. New algorithms to adapt the physical representation to new architectures will be required.

The "great observatories" promise to revolutionize our view of the universe. Numerical and theoretical advances are vital so that we can intelligently interpret the new observational advances and make theoretical predictions to use the observational resources efficiently.

\section{Conclusions}

The vantage point of doing science from space has greatly expanded the role and importance of computer simulation and modeling research in the space and earth sciences. The global views of the earth or the panoramic views of the universe that remote sensing provides generate massive data streams of scientific information on the existence, evolution, and dynamic behavior of complex interactive systems.

From the examples of simulation studies presented in Section II we have seen that current numerical models are proving essential for understanding the science, but equally 
important is their use as tools for assimilating and validating data.

The most striking theme to emerge across the varied scientific discipline problems represented in Section III is the uniform desire by scientists to construct hybrid codes linking models with different spatial, temporal, and physical processes into one larger system. Geophysicists are attempting to model the entire solid earth from core to mantle, and atmospheric scientists are starting to model the global ocean-land-biological-chemical dynamic processes. Solar physicists are studying the coupling of the deep interior with processes on the surface of the sun. Stellar and cosmic scientists are expanding their studies of the evolution of stars and galaxies to include their interaction with surrounding clouds of gas. Space observations have been the major motivating force in driving these studies into these new directions. However, the research efforts would not have been possible without the accompanying increase in computational power. Future interdisciplinary research programs have some very unique requirements if they are to attain the same levels of success met by the discipline models.

Based on estimates derived from current computing systems for several of the individual models described, the projected requirements for the interdisciplinary modeling studies are shown in Fig. 25. The requirements for these scientific challenges call for computational speeds in excess of one teraflop and for memory requirements in excess of a teraword. It is clear that if computer systems are going to provide scientists with a thousandfold increase in speed and memory in the next decade, it will only come about through the development of parallel processing architectures.

Fortunately for these scientific problems, parallel architectures are ideally suited to them. Most components of these hybrid systems involve Navier-Stokes dynamics, which are themselves highly vectorizable or parallelizable. As for computing the different scientific components, they can probably be executed concurrently in different processors and then synchronized. While this mapping seems simple and natural, it will involve a serious rethinking and restructuring of systems that have been developed over decades for optimal performance on classical von Neumann architectures. Thus, a major computer science resource effort to optimize algorithms for these new computer systems will have to be planned as well. Significant progress on such problems will not occur without significant improvements in hardware and software systems, algorithms, and peripherals.

In addition, the challenge in the earth sciences is different in one major respect from that in space sciences in that while the space science data volumes are on the order of 1 Tbyte per year, the earth science data are expected to be on the order of 1 Tbyte per day. Thus, in addition to supercomputers, massive high-speed data storage and rapid input/output access capability, on the order of $10^{18}$ bytes, is equally critical.

Such computational requirements appear within technological reach in the next decade if a dedicated national commitment is made to encourage their development. Certainly, the prediction and understanding of processes such as the following offer grand scientific challenges, worth making such a commitment:

- Greenhouse effect

- Ozone hole

- El Niño

- Earthquakes and geomagnetism

- Interior thermal structure of the sun

- Solar-terrestrial effects

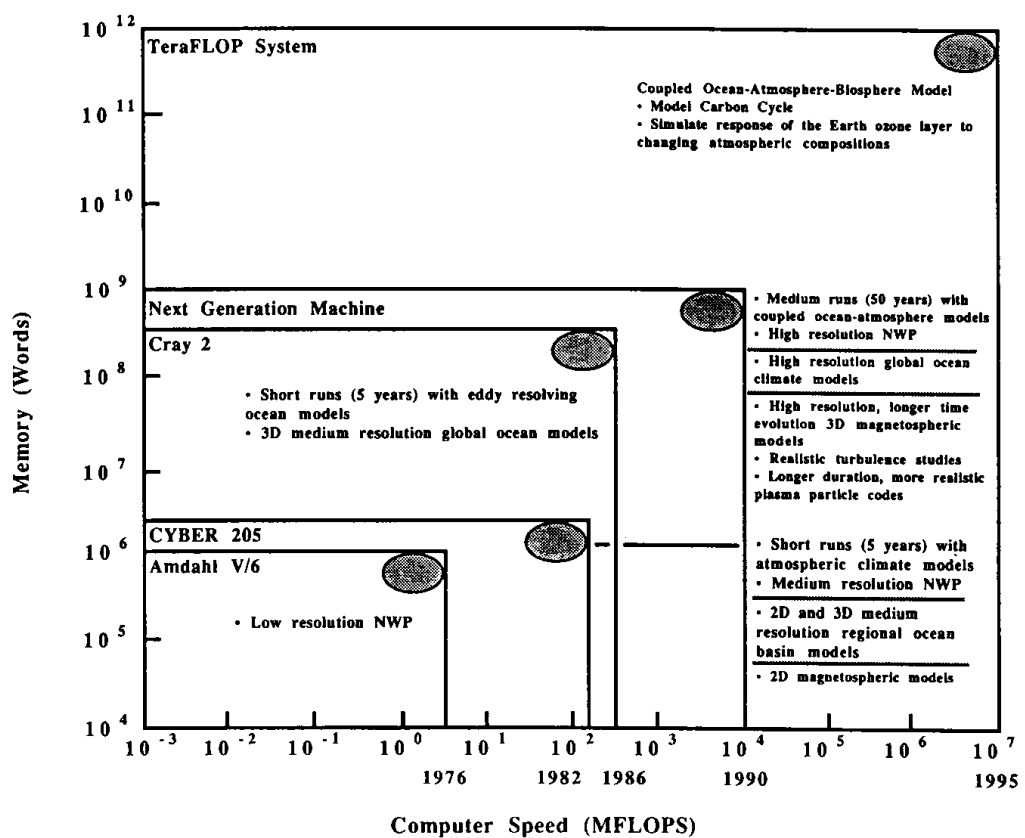

Fig. 25. Projected computing needs for space and earth science modeling and simulation. 
- Supernovas and galaxies

- Origin of the universe

- Origin of life

The benefits will not only yield an improved fundamental understanding of earth and space sciences, but possibly the ability to better manage humankind's activities and adverse influence on the environment.

\section{ACKNOWLEDGMENT}

Without the contributions of leading scientists in their respective fields it would not have been possible to describe forefront results from such a broad range of scientific disciplines as reported in this paper. Their research and ideas have greatly influenced the tone of this survey paper in space and earth science modeling. In particular, the author wishes to acknowledge C. Voorhies, P. Schopf, M. Suarez, C. Parkinson, Y. Sud, R. Rood, J. Kaye, D. Spicer, P. MacNeice, J. Blondin, J. Strong, H. Ramapriyan, J. Dorband, and J. Hollis. He is especially grateful to Mark Stevens and Mary Ford for their unstinting dedication of time and energy in providing all the exceptional technical and editorial assistance without which this paper could not have been completed in time for this issue.

\section{REFERENCES}

[1] C. V. Voorhies, "Steady flows at the top of earth's core derived from geomagnetic field models," J. Ceophys. Res., vol. 91, pp. 444-466, 1986.

[2] - "Steady surficial core motions: An alternate method," Ceophys. Res. Lett., vol. 13, pp. 1537-1540, 1986.

[3] - "Joint inversion of geophysical data (MERGES)," in Proc. 1st SEDI Symp., p. 39, 1988.

[4] P. H. Roberts and S. Scott, "On analysis of secular variation 1: A hydrodynamic constraint," J. Geomag. Geoelectr., vol. 17, pp. 137-151, 1965.

[5] C. V. Voorhies and G. E. Backus, "Steady flows at the top of earth's core from geomagnetic field models: The steady motion theorem," Geophys. Astrophys. Fluid Dyn., vol. 32, pp. $163-173,1985$.

[6] C. V. Voorhies, "Probing surface core motions with DGRF models," EOS, vol. 69, no. 16, Apr. 1988.

[7] R. A. Langel, D. R. Varraclaugh, D. J. Kerridge, V. P. Gilokov, T. J. Sabaka, and R. H. Estes, "Definitive IGRF models for 1945, 1950, 1955, and 1960,"J. Geomag. Geoelectr., vol. 40, pp.645702, 1988.

[8] P. S. Schopf, "Simulating the annual cycle of the tropical Pacific with a hybrid coordinate upper ocean model,"J. Geophys. Res. (in press).

[9] H. Peters, M. C. Gregg, and J. M. Toole, "On the parameterization of equatorial turbulence," $J$. Geophys. Res., vol. 93 , pp. 1199-1218, 1988.

[10] R. Legeckis, W. Pickely, and G. Nesterczuk, Bull. Am. Met. Soc., vol. 64, pp. 133-139, 1983.

[11] S. Levitus, "Climatological atlas of the world ocean," NOAA Prof. Paper 13, 173, 1982.

[12] K. Wyrtki and B. Kilonsky, "Mean water and current structure during the Hawaii to Tahiti shuttle experiment," J. Phys. Oceanogr., vol. 14, pp. 242-254, 1984.

[13] K. Wyrtki and W. G. Leslie, "The mean annual variation of sea level in the Pacific Ocean," Hawaii Inst. of Geophys., Univ. of Hawaii, Honolulu, Rep. HIG-80-5, 159 pp.

[14] A. Arakawa and M. Suarez, 1981.

[15] R. Harshvardhan, D. A. Davies, and T. G. Corsetti, "A fast radiation parameterization for atmospheric circulation models," J. Geophys. Res., vol. 92, pp. 1009-1016, 1987.

[16] M.D. Chou, "Broadband water vapor transmission functions for atmospheric IR flux computations," J. Atmos. Sci., vol. 41, pp. $1775-1778,1984$.

[17] M.D. Chou and L. Peng, "A parameterization of the absorption in the $15 \mu \mathrm{m} \mathrm{CO}$ spectral region with application to cli- mate sensitivity studies," J. Atmos. Sci., vol. 40, pp. 2183-2192, 1983.

[18] C. D. Rodgers, "Some extensions and applications of the new random model for molecular band transmission," $Q$. J. $R$. Meteorol. Soc., vol. 94, pp. 99-102, 1968.

[19] J. E. Rosenfield, M. R. Schoeberl, and M. A. Geller, "A computation of the stratospheric diabatic circulation using an accurate radiative transfer model," J. Atmos. Sci., vol. 44, pp. 859-876, 1987

[20] A. Arakawa and W. Schubert, "Interaction of a cumulus cloud ensemble with the large-scale environment," J. Atmos. Sci. vol. 31, pp. 674-701, 1974.

[21] C. L. Parkinson and W.M. Washington, "A large-scale numerical model of sea-ice," J. Geophys. Res., vol. 84, pp. 311-337, 1979.

[22] S. Hakkinen, "A coupled dynamic-thermodynamic ice ocean model for the marginal ice zone," J. Ceophys. Res., vol. 92, pp. 9469-9478, 1987.

[23] S. Hakkinen and D. J. Cavalieri, "A study of surface heat fluxes in the Norwegian Greenland and Barents Sea," J. Geophys. Res., 1988 (submitted for publication).

[24] P. Sellers, Y. Mintz, Y. Sud, and A. Dalcher, "A simple biosphere model $(\mathrm{SiB})$ for use within general circulation models," J. Atmos. Sci., vol. 43, pp. 505-531, 1986.

[25] M. Halem, "Applications of remote image sensing with the NASA massively parallel processor," Phil. Trans. R. Soc. London, $A$, vol. 324, pp. 365-372, 1988.

[26] J. L. Potter, Ed., The Massively Parallel Processor. Cambridge, MA: M.I.T. Press, 1985.

[27] K. E. Batcher, "Design of a massively parallel processor," IEEE Trans. Comput., vol. C-29, pp. 836-840, 1980.

[28] D. Marr and T. Poggio, "A computational theory of human stereo vision," Proc. R. Soc., pp. 301-328, 1979.

[29] L. Quam, "Hierarchical warp stereo," in Proc. Image Understanding Workshop, pp. 149-155, 1984.

[30] J. Dorband, "3-D graphics generation on the MPP," in Proc. 2nd Int. Conf. on Supercomputing, pp. 305-309, 1986.

[31] J. P. Strong and H. K. Ramapriyan, "Massively parallel correlation techniques to determine local differences in pairs of images," in Proc. 2nd Int. Conf. on Supercomputing, 1987.

[32] R. B. Rood, D. J. Allen, W. E. Baker, D. J. Lamich, and J. A. Kaye "The use of assimilated stratospheric data in constituent transport experiments," J. Atmos. Sci., 1989 (in press).

[33] R. B. Rood, J. A. Kaye, J. E. Nielsen, M. R. Schoeberl, and M. A. Geller, "Nitric acid forecast experiments," Phys. Scripta, vol. 36, pp. 337-354, 1987.

[34] I. A. Kaye and R. B. Rood, "Chemistry and transport in a threedimensional stratospheric model: Chlorine species during simulated stratospheric warming," J. Geophys. Res., 1989 (in press).

[35] H. Alfven and P. Carlquist, Solar Phys., vol. 1, p. 220, 1967.

[36] R. Rosner, W. H. Tucher, and G. S. Vaiana, Astrophys. J., vol. 220 , p. $643,1978$.

[37] D. S. Spicer, Solar Phys., vol. 70, p. 149, 1981.

[38] _- Space Sci. Rev., p. 351, 1982.

[39] G. Benford, Astrophys. J., vol. 269, p. 690, 1983.

[40] G. Holman, Astrophys. J., vol. 293, p. 584, 1985.

[41] G. Van Hoven, in Solar Flare Magnetohydrodynamics, E. R. Priest, Ed. London, England: Gordon and Breach, 1981, chap. 4.

[42] E. R. Priest, Ed., Solar Flare Magnetohydrodynamics. London, England: Gordon and Breach, 1981, chap. 3.

[43] R. W. Hockney and J. W. Eastwood, Computer Simulation Using Particles. New York, NY: McGraw-Hill, 1982.

[44] C. K. Birdsall and A. B. Langdon, Plasma Physics via Computer Simulation. New York, NY: McGraw-Hill, 1985.

[45] T. Northrop, "The adiabiatic motion of charged particles," Tract, Interscience, New York, 1963.

[46] S. Chandrasekhar, Astrophys. J., vol. 97, p. 255, 1943.

[47] L. Spitzer, Physics of Fully lonized Gases. New York, NY: Wiley, 1962

[48] A. Koenigl, in Proc. 12th Texas Symp. on Relativistic Astrophysics, New York Academy of Sciences, vol. 470, pp. 88-105 1986.

[49] T. Buehrke, R. Mundt, and T.P. Ray, "A detailed study of HH34 and its associated jet," Astron. Astrophys., vol. 200, pp. 99$119,1988$. 
[50] I. M. Blondin, A. Koenigl, and B. A. Fryxell, "Herbig-Haro objects as the heads of radiative jets," Astrophys. J. Lett., 1989.

[51] P. Collela and P. R. Woodward, J. Comp. Phys., vol. 54, p. 174, 1984.

[52] D. E. Innes, J. R. Giddings, and Sage Falle, Mon. Not. R. Astron. Soc., p. 226, 1987.

[53] M. Kafatos, Astrophys. J., vol. 182, p. 433, 1973.

[54] D. L. T. Anderson and J. P. McCreary, "Slowly propagating disturbances in a coupled ocean atmosphere model," $j$. Atmos. Sci., vol. 42, pp. 615-629, 1985.

[55] A. J. Hirst, "Slow instabilities in tropical ocean basin-global atmosphere models," J. Atmos. Sci., vol. 45, pp. 830-852, 1987.

[56] M. J. Suarez and P. S. Schopf, "A delayed-action oscillator for ENSO" I. Atmos. Sci., 1988 (in press).

[57] M. A. Cane and S. E. Zebiak, "A theory for El Nino and the southern oscillation," Science, vol. 228, pp. 1085-1087, 1985.

[58] G. A. Maykut, "Energy exchange over young sea ice in the central Arctic," I. Geophys. Res., vol. 83, pp. 3646-3658, 1978.

[59] J. J. Zwally, J. C. Comiso, C. L. Parkinson, W. J. Campbell, F. D. Carsey, and P. Gloersen, "Antarctic sea ice, 1973-1976: Satellite passive-microwave observations," National Aeronautics and Space Administration, Rep. NASA SP-459, 1983.

[60] C. L. Parkinson, J. C. Comiso, J. H. Zwally, D. J. Cavalieri, P. Gloersen, and W. J. Campbell, "Arctic sea ice, 1973-1976: Satellite passive microwave observations," National Aeronautics and Space Administration, Rep. NASA-SP 489, 1987.

[61] D. J. Cavalieri, P. Gloersen, and W. J. Campbell, "Determination of sea ice parameters with the Nimbus-7 SMMR," J. Geophys. Res., vol. 89, pp. 5355-5369, 1984.

[62] R. W. Eppley, "New production: History, methods, problems," in Proc. Dahlem Conf. Workshop on Production of Oceans: Past and Present, 1988.

[63] C. K. Stidd, "Local moisture and precipitation," Desert Research Inst., Univ. of Nevada, Preprint 45a, 1968.

[64] J. McDonald, "The evaporation-precipitation fallacy," Weather, pp. 168-177, 1962.

[65] M. Budyko, Climate and Life, D. H. Miller, Transl. New York NY: Academic Press, 1974.

[66] P. S. Eagleson, "The emergence of global-scale hydrology," Water Resour. Res., vol. 27, pp. 6S-14S, 1986.

[67] T. C. Yeh, R. T. Wetherald, and S. Manabe, "The effect of soil moisture on the short-term climate and hydrology changeA numerical experiment," Mon. Weather Rev., vol. 112, pp. 474-490, 1984.
[68] J. Walker and P. R. Rowntree, "The effect of soil moisture on circulation and rainfall in a tropical model," Q. J. R. Meteorol. Soc., vol. 103, p. 2946, 1977.

[69] J. Shukla and Y. Mintz, "Influence of land-surface evapotranspiration on the earth's climate," Science, vol. 215, pp. 1498-1501, 1982.

[70] D. Rind, "The influence of vegetation on the hydrological cycle in a global climate model," Geophys. Mono. 29, Maurice-Ewing, vol. 5, pp. 73-91, 1984.

[71] U. C. Sud and M. J. Fennessy, "Influence of evaporation in semi-arid regions on the July circulation: A numerical study," J. Climatol., vol. 4, pp. 383-398, 1984.

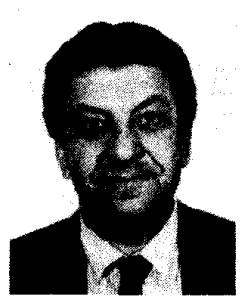

Milton Halem was born in Brooklyn, NY. He received the Bachelor's degree from City College, New York, and the Ph.D. degree in 1968 from the Courant Institute of Mathematical Sciences, New York University, New York, both in mathematics.

He joined NASA in 1971 as a Senior Mathematical Analyst with the Goddard Institute for Space Studies in New York. In 1977 he came to Goddard Space Flight Center (GSFC) in Washington, DC, where he served as GARP Project Scientist and later as Head of the Global Modeling and Simulation Branch. Currently he is Chief of the Space Data and Computing Division at GSFC in Greenbelt, MD. Under his leadership GSFC developed one of the world's foremost atmospheric general circulation models leading to major scientific contributions in assessing and improving the impact of satellite observing systems for weather forecasting and for establishing the physical basis for climate prediction. He was instrumental in bringing to GSFC a CYBER 205, which along with the massively parallel processor and several advanced IBM processors and peripherals comprises one of the world's most powerful scientific computing complexes.

Dr. Halem has published extensively on atmospheric and oceanographic sciences, in particular on his pioneering research in simulating the forecast impact of space observing systems. He received the Goddard Exceptional Performance Award in 1974 and 1978 , the NASA Medal for Exceptional Scientific Achievement in 1979, the NASA Certificate for Outstanding Performance in 1982, and a number of peer awards. 